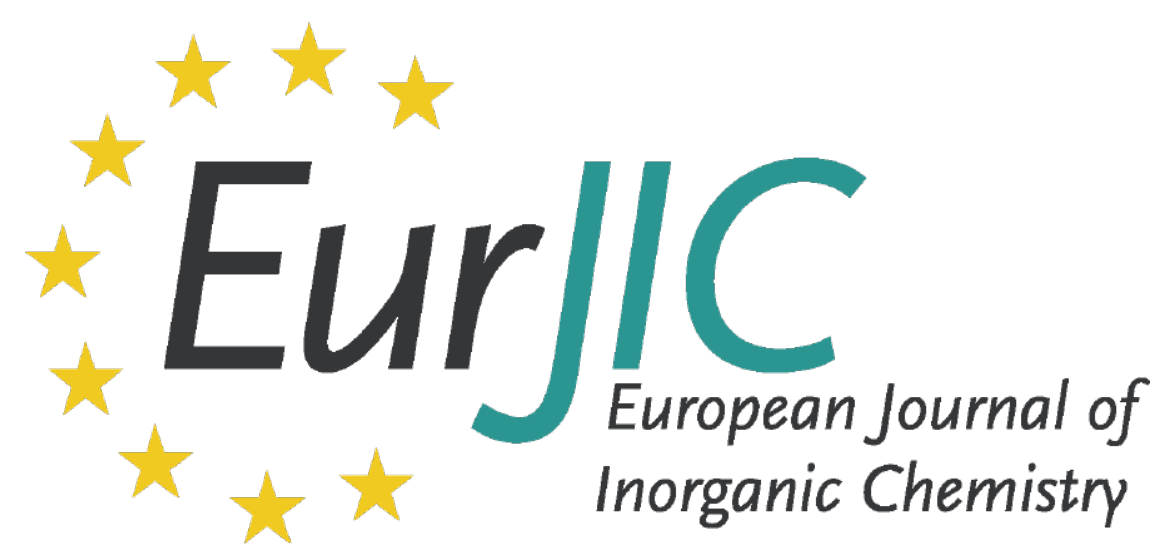

Supporting Information

\title{
A Dicationic Bismuth(III) Lewis Acid: Catalytic Hydrosilylation of Olefins
}

Selvakumar Balasubramaniam, Sandeep Kumar, Alex P. Andrews, Babu Varghese, Eluvathingal D. Jemmis, and Ajay Venugopal*

ejic201900459-sup-0001-SupMat.pdf 


\section{Contents}

1. Experimental Methods

2. Synthesis and characterization

3. Modified Gutmann Test

4. Catalytic Hydrosilylation

5. Crystallographic Details

6. Computational Details

7. References 


\section{Experimental Methods:}

All manipulations were performed under argon atmosphere using standard Schlenk and glove-box techniques. $^{[1]}$ Solvents used for the synthesis and NMR experiments were dried, distilled and degassed before use by standard methods. ${ }^{[2]}$ Anhydrous $\mathrm{BiCl}_{3}$ was purchased from Sigma-Aldrich. The starting materials potassium hydridotris(3,5-dimethylpyrazolyl)borate (KTp*) ${ }^{[3]}$ and triethylsilyliumtetrakis(pentafluorophenyl)borate $\left[\mathrm{Et}{ }_{3} \mathrm{Si}\right]\left[\mathrm{B}\left(\mathrm{C}_{6} \mathrm{~F}_{5}\right)_{4}\right]^{[4]}$ were prepared according to the literature procedure. NMR measurements were performed on Bruker $500 \mathrm{MHz}$ spectrometer. The chemical shifts ( $\delta \mathrm{ppm})$ in ${ }^{1} \mathrm{H}$ NMR spectra were referenced to the residual proton signals in the deuterated solvents. The chemical shifts $(\delta \mathrm{ppm})$ in ${ }^{11} \mathrm{~B}$ NMR spectra were referenced to $\mathrm{NaBH}_{4}$ in $\mathrm{D}_{2} \mathrm{O}$. The chemical shifts $(\delta \mathrm{ppm})$ in ${ }^{19} \mathrm{~F}$ NMR spectra were referenced to $\mathrm{CFCl}_{3}$. Samples for elemental analysis, dried at $1 \times 10^{-3} \mathrm{mbar}$ at ambient temperature, packed under inert conditions were analysed using Elementar Vario Micro Cube instrument. 


\section{Synthesis and Characterization}

Compound 1: Toluene $(50 \mathrm{~mL})$ was condensed to a mixture of $\mathrm{KTp}^{\mathrm{Me2}}(1 \mathrm{mmol}, 0.336 \mathrm{~g})$ and $\mathrm{BiCl}_{3}(1$ $\mathrm{mmol}, 0.315 \mathrm{~g}$ ) at $-196{ }^{\circ} \mathrm{C}$. The reaction mixture was allowed to warm to ambient temperature and stirred for 12 hours. Toluene was evaporated under vacuum resulting colorless powder, which was mixed with $\mathrm{B}\left(\mathrm{C}_{6} \mathrm{~F}_{5}\right)_{3}(2 \mathrm{mmol}, 1.024 \mathrm{~g})$. The mixture was extracted with $30 \mathrm{~mL}$ of $\mathrm{CH}_{2} \mathrm{Cl}_{2}$ and filtered to separate $\mathrm{KCl}$. The filtrate was concentrated and layered with $\mathrm{n}$-pentane at room temperature. After 7 days, colorless crystals of 1 were obtained (yield $80 \%, 0.460 \mathrm{~g}) ;{ }^{1} \mathrm{H} \mathrm{NMR}\left(\mathrm{CD}_{2} \mathrm{Cl}_{2}, 500 \mathrm{MHz}\right): \delta 6.01$ (s, 3H, CH ), ठ $4.73(\mathrm{~s}(\mathrm{br}), 1 \mathrm{H}, \mathrm{BH}), \delta 2.58(\mathrm{~s}, 9 \mathrm{H}, \mathrm{CH} 3), \delta 2.41$ (s, 9H, CH3); ${ }^{11} \mathrm{~B} \mathrm{NMR}\left(\mathrm{CD}_{2} \mathrm{Cl}_{2}, 160\right.$ $\mathrm{MHz}): \delta-4.69$ (s(br), $\mathrm{BH}) ;{ }^{13} \mathrm{C} \mathrm{NMR}\left(\mathrm{CD}_{2} \mathrm{Cl}_{2}, 125 \mathrm{MHz}\right): \delta 152.1\left(\mathrm{CH}_{3} \mathrm{CNN}\right), \delta 146.6\left(\mathrm{CH}_{3} \mathrm{CNB}\right), \delta$ $107.6(\mathrm{CH}), \delta 14.2\left(\mathrm{CH}_{3} \mathrm{CNB}\right), \delta 12.6\left(\mathrm{CH}_{3} \mathrm{CNN}\right)$; Elemental analysis: calculated for $\mathrm{C}_{15} \mathrm{H}_{22} \mathrm{~N}_{6} \mathrm{BBiCl}_{2}$ : C 31.2 H 3.8 N 14.5 Found C 31.4 H 3.5 N 14.5.
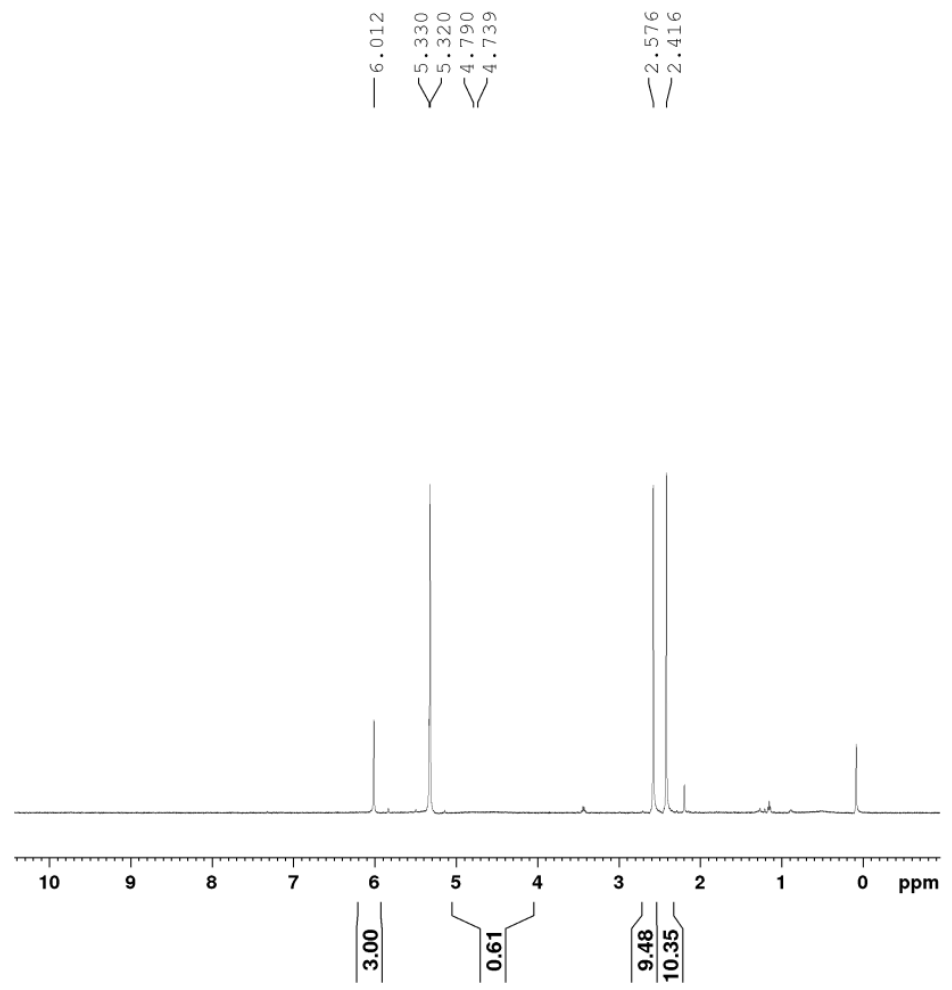

Figure S1: ${ }^{1} \mathrm{H}$ NMR spectrum of 1 in $\mathrm{CD}_{2} \mathrm{Cl}_{2}$. 


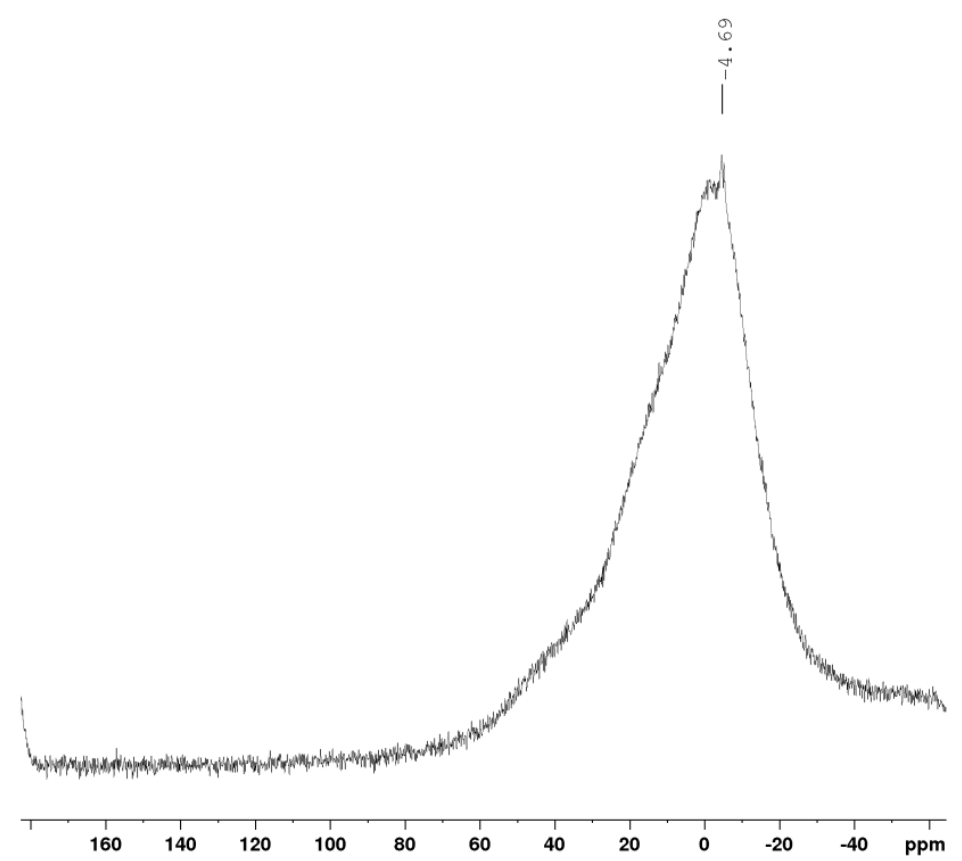

Figure S2: ${ }^{11} \mathrm{~B}$ NMR spectrum of 1 in $\mathrm{CD}_{2} \mathrm{Cl}_{2}$.

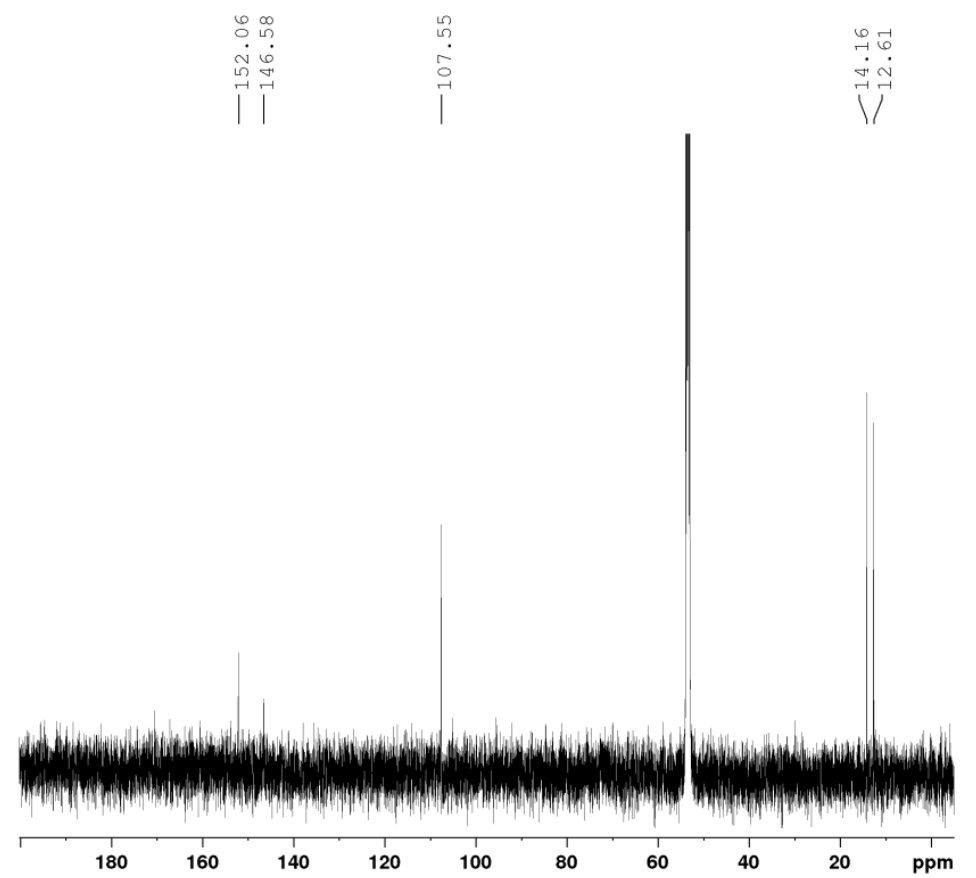

Figure S3: ${ }^{13} \mathrm{C}$ NMR spectrum of 1 in $\mathrm{CD}_{2} \mathrm{Cl}_{2}$. 
Compound 2: Ortho-dichlorobenzene $(10 \mathrm{~mL})$ was added to a mixture of $\mathrm{Tp}^{\mathrm{Me} 2} \mathrm{BiCl}_{2}(0.17 \mathrm{mmol}, 0.100$ g) and $\left[\mathrm{Et}_{3} \mathrm{Si} .\left(\mathrm{C}_{9} \mathrm{H}_{12}\right)\right]\left[\mathrm{B}\left(\mathrm{C}_{6} \mathrm{~F}_{5}\right)_{4}\right](0.35 \mathrm{mmol}, 0.320 \mathrm{~g})$ at room temperature. The reaction mixture was allowed to stir for two hours. A pale yellow solution was obtained. $n$-Pentane $(30 \mathrm{~mL})$ was added to the reaction mixture with vigorous stirring. An oily precipitate separated from mother liquor and it was dried under vacuum to obtain a colorless precipitate of 2 (Yield 70\%, $0.240 \mathrm{~g}$ ). 2 was crystallized from a mixture of ortho-dichlorobenzene and chlorobenzene at room temperature after several days. ${ }^{1} \mathrm{H}$ NMR (ortho-dichlorobenzene-D4, $500 \mathrm{MHz}$ ): $\delta 6.35$ (s, 3H, CH ), ठ 4.89 (s(br), 1H, BH), ठ 2.63 (s, 9H, $\mathrm{CH} 3$ ), $\delta 2.51$ (s, 9H, CH3); ${ }^{11} \mathrm{~B}$ NMR (ortho-dichlorobenzene-D4, $160 \mathrm{MHz}$ ): $\delta\left(\mathrm{s},-13.53, \mathrm{~B}\left(\mathrm{C}_{6} \mathrm{~F}_{5}\right)_{4}\right)$; ${ }^{13} \mathrm{C}$ NMR (ortho-dichlorobenzene-D4, $\left.125 \mathrm{MHz}\right)$ : $\delta 155.1\left(\mathrm{CH}_{3} \mathrm{CNN}\right.$ ), $\delta 153.5\left(\mathrm{CH}_{3} \mathrm{CNB}\right.$ ), $\delta 153.5$ (o$\left.\mathrm{CF}-\left[\mathrm{B}\left(\mathrm{C}_{6} \mathrm{~F}_{5}\right)\right]_{4}\right), \delta 137.4 \mathrm{ppm}\left(p-\mathrm{CF}-\left[\mathrm{B}\left(\mathrm{C}_{6} \mathrm{~F}_{5}\right)\right]_{4}\right), \delta 135.5$ ppm $\left(m-\mathrm{CF}-\left[\mathrm{B}\left(\mathrm{C}_{6} \mathrm{~F}_{5}\right)\right]_{4}\right), \delta 123.9$, (ipso-C - $\left.\left[\mathrm{B}\left(\mathrm{C}_{6} \mathrm{~F}_{5}\right)\right]_{4}\right), \delta 111.1(\mathrm{CH}), \delta 12.9\left(\mathrm{CH}_{3} \mathrm{CNB}\right), \delta 12.3\left(\mathrm{CH}_{3} \mathrm{CNN}\right),{ }^{19} \mathrm{~F}$ NMR (ortho-dichlorobenzene-D4, $470.58 \mathrm{MHz}): \delta\left(\mathrm{s},-131.52\right.$, o-CF - $\left.\left[\mathrm{B}\left(\mathrm{C}_{6} \mathrm{~F}_{5}\right)\right]_{4}\right), \delta\left(\mathrm{s},-165.45\right.$, m-CF - $\left.\left[\mathrm{B}\left(\mathrm{C}_{6} \mathrm{~F}_{5}\right)\right]_{4}\right), \delta(\mathrm{s},-161.26, p-\mathrm{CF}-$ $\left.\left[\mathrm{B}\left(\mathrm{C}_{6} \mathrm{~F}_{5}\right)\right]_{4}\right)$. Elemental analysis: calculated for $\mathrm{C}_{69} \mathrm{H}_{27} \mathrm{~N}_{6} \mathrm{~B}_{3} \mathrm{~F}_{40} \mathrm{Cl}_{1} \mathrm{Bi}_{1}$ : $\mathrm{C} 41.9 \mathrm{H} 1.4 \mathrm{~N} 4.2$ Found $\mathrm{C} 42.0$ H 1.2 N 4.0.

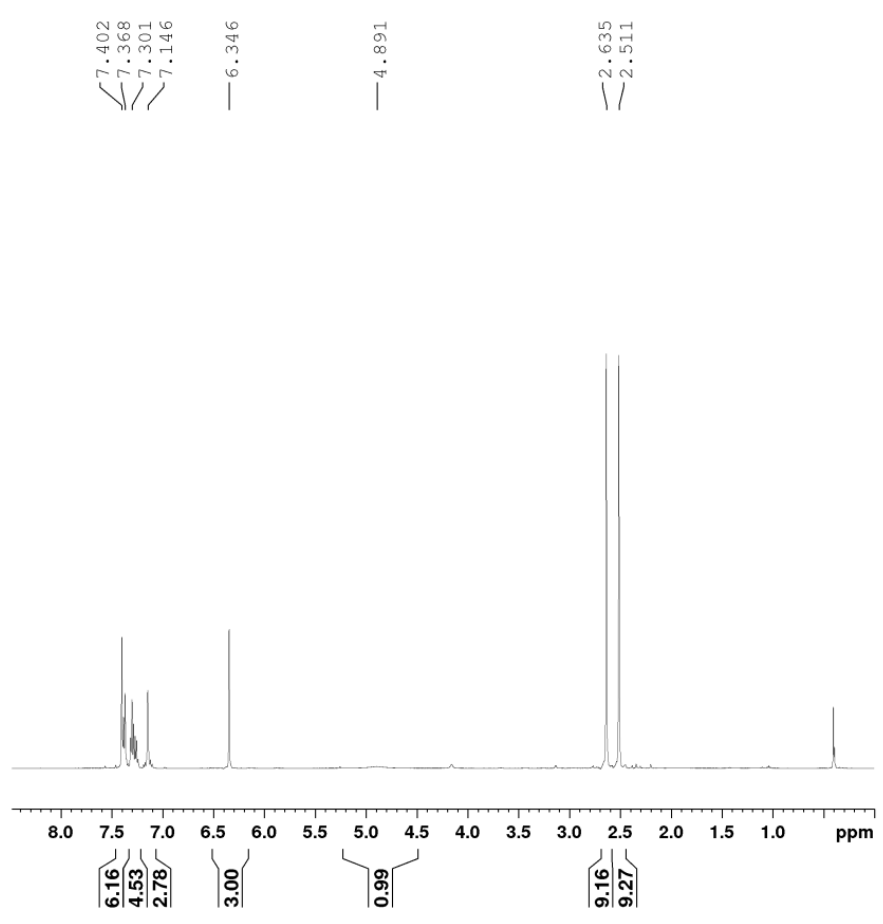

Figure S4: ${ }^{1} \mathrm{H}$ NMR spectrum of 2 in ortho-dichlorobenzene-D4. 


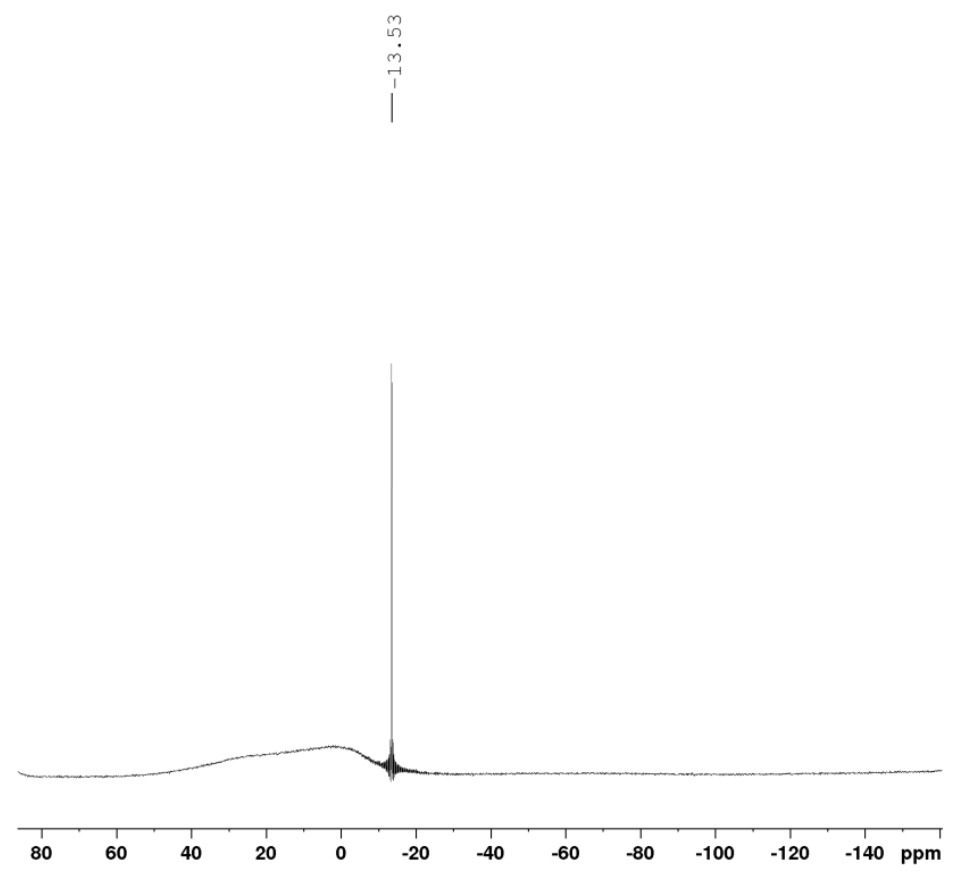

Figure S5: ${ }^{11} \mathrm{~B}$ NMR spectrum of 2 in ortho-dichlorobenzene-D4.

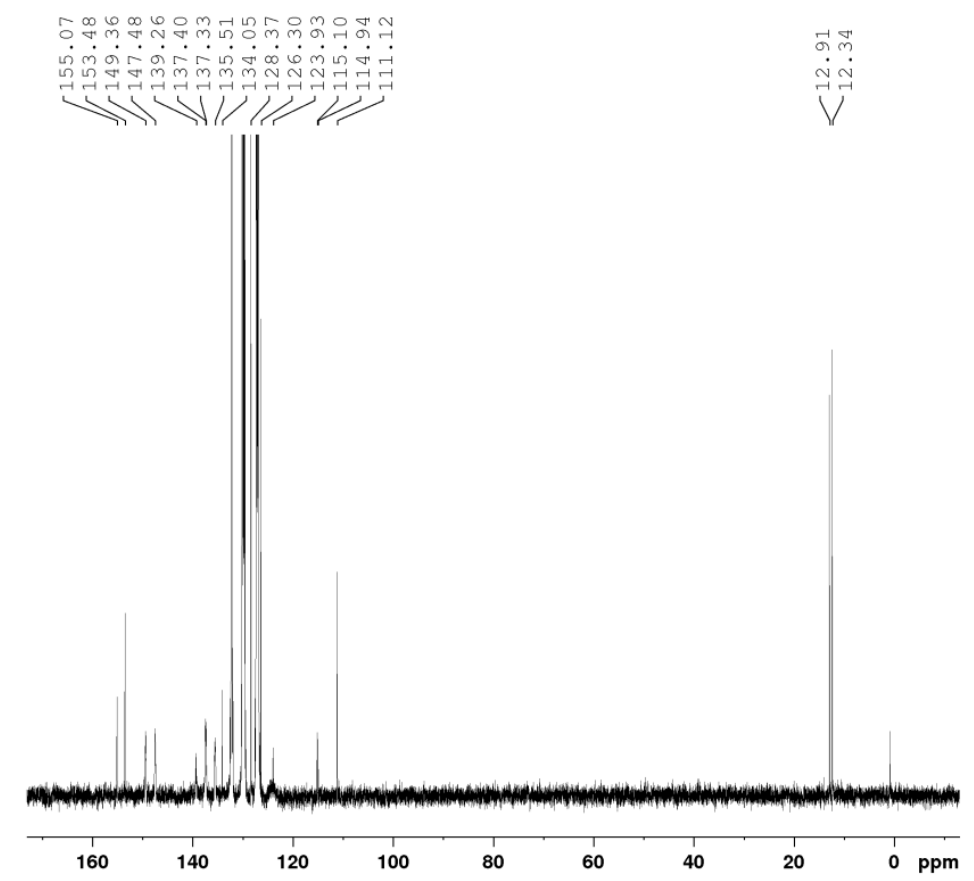

Figure S6: ${ }^{13} \mathrm{C}$ NMR spectrum of 2 in ortho-dichlorobenzene-D4. 


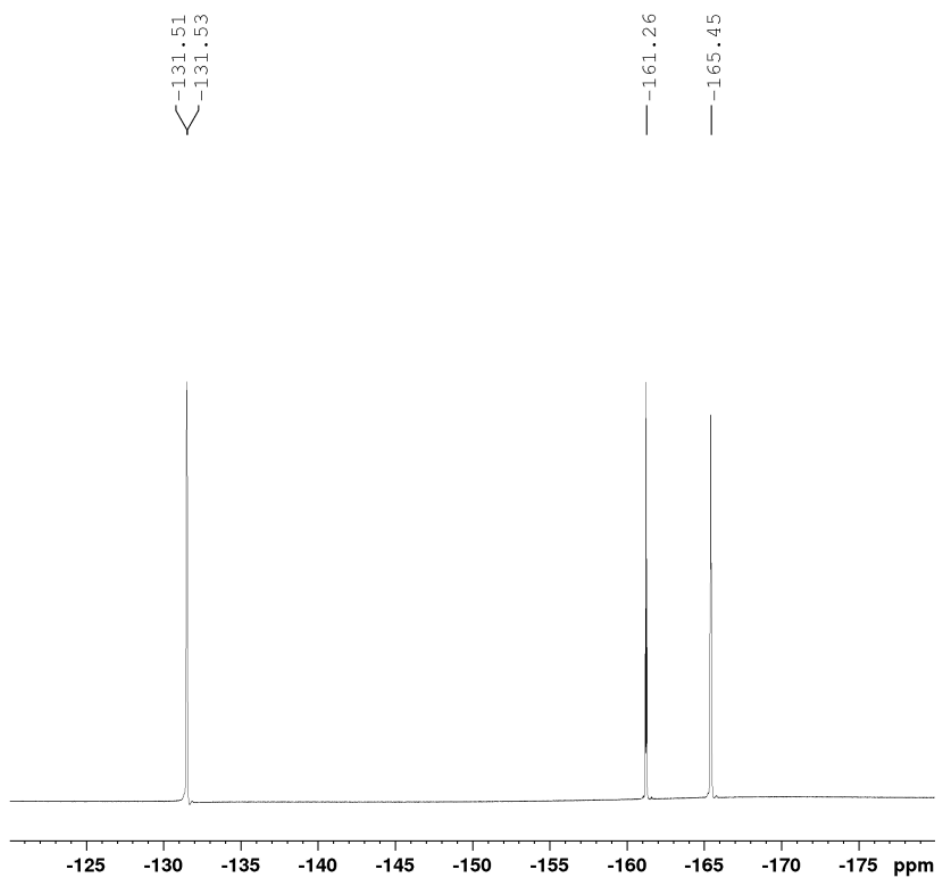

Figure S7: ${ }^{19} \mathrm{~F}$ NMR spectrum of 2 in ortho-dichlorobenzene-D4. 
3. Modified Gutmann Test:

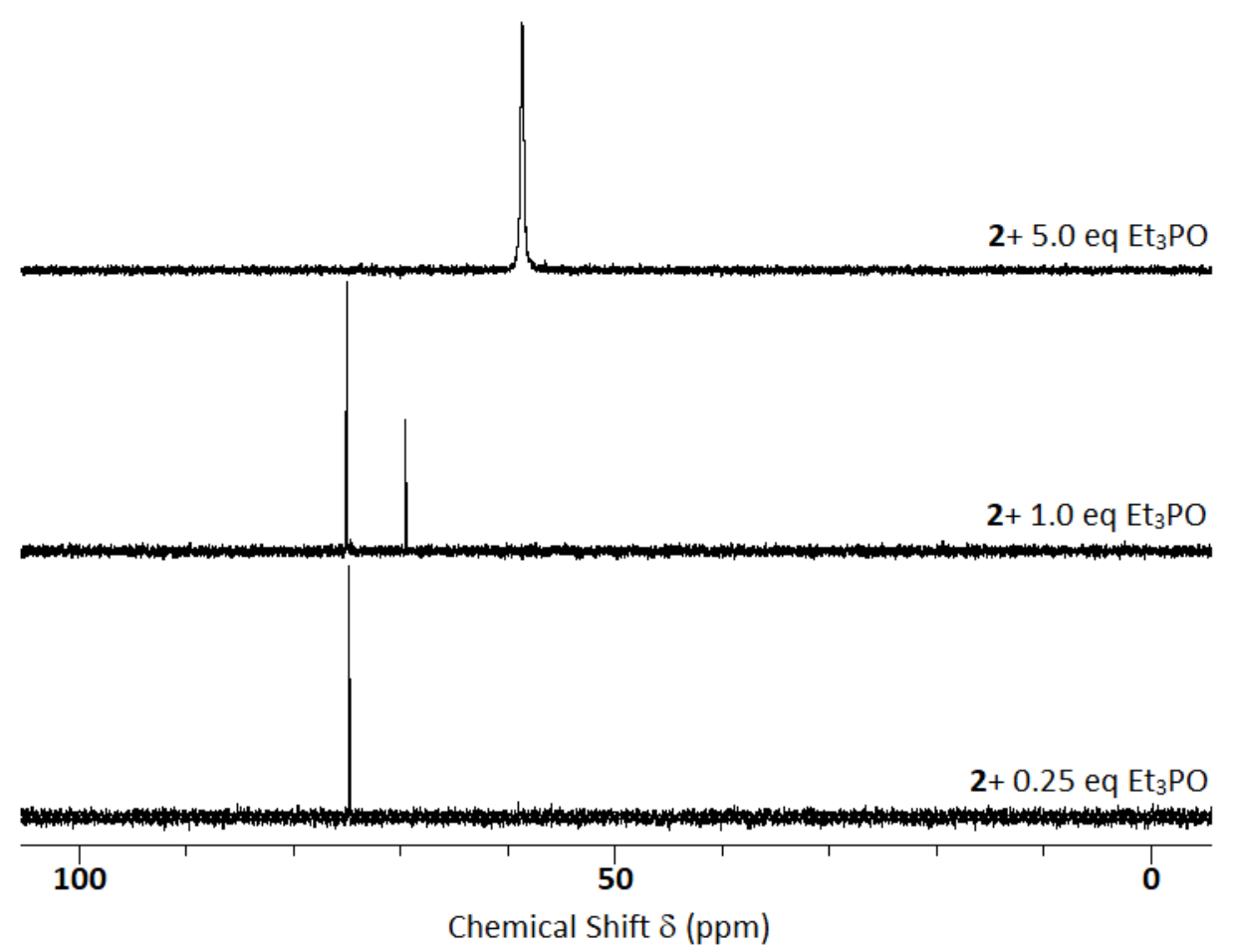

Figure S8: In situ reaction between 2 and $\mathrm{Et}_{3} \mathrm{PO}$ in ortho-dichlorobenzene-D4 as monitored using ${ }^{31} \mathrm{P}$ NMR spectroscopy.
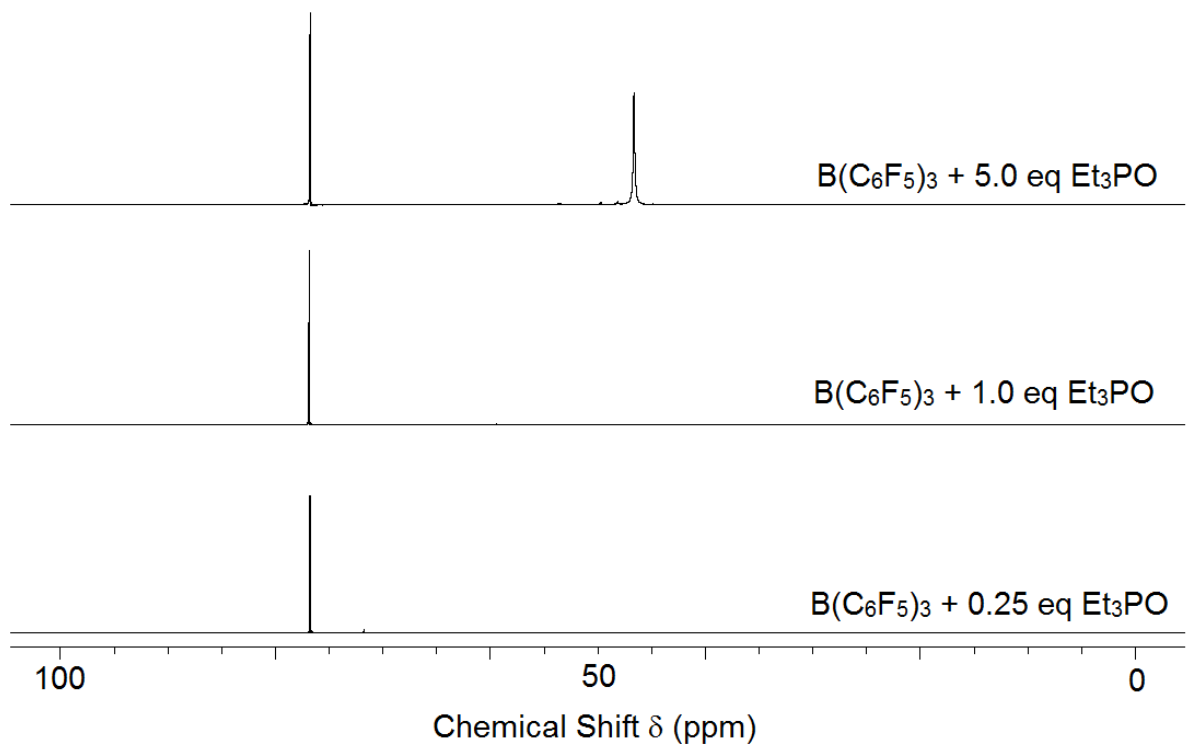

Figure S9: In situ reaction between $\mathrm{B}\left(\mathrm{C}_{6} \mathrm{~F}_{5}\right)_{3}$ and $\mathrm{Et}_{3} \mathrm{PO}$ in ortho-dichlorobenzene-D4 as monitored using ${ }^{31} \mathrm{P}$ NMR spectroscopy. 


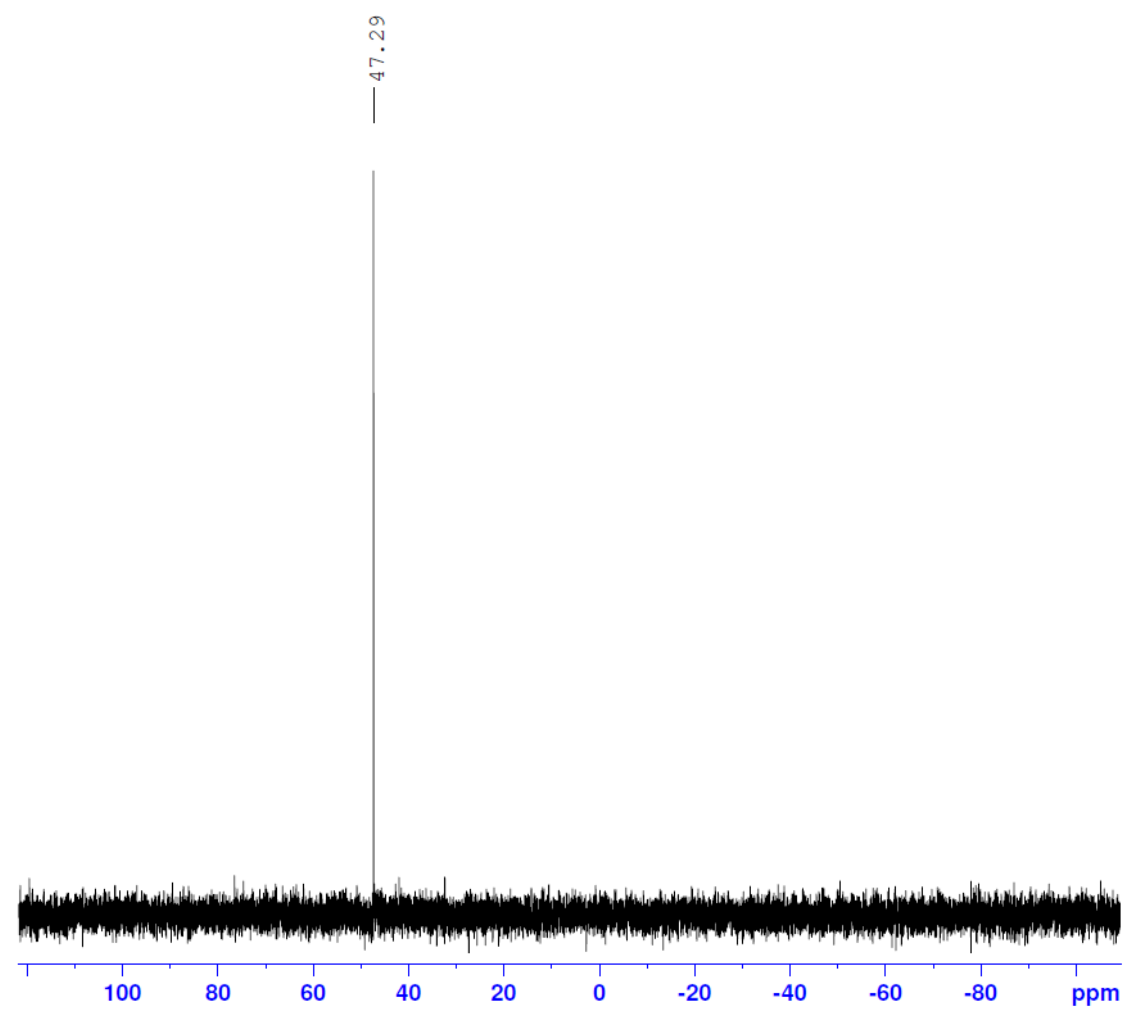

Fig S10: ${ }^{31} \mathrm{P}$ NMR spectrum of triethylphosphine oxide in ortho-dichlorobenzene-D4 


\section{Catalytic Hydrosilylation}

Typical procedure: $1 \mathrm{mmol}$ of triethylsilane $(0.158 \mathrm{ml})$ and $1 \mathrm{mmol}$ of olefin were mixed in a J-young nmr tube followed by the addition of $0.6 \mathrm{ml}$ of ortho-dichlorobenzene- ${ }_{4}{ }_{4}{ }^{1} \mathrm{H}$ NMR spectrum of the solution was recorded $0.001 \mathrm{mmol}$ of compound 2 was added to this solution at $20^{\circ} \mathrm{C}$ and the completion of hydrosilylation was confirmed by NMR spectroscopy. $0.1 \mathrm{mmol}$ of mesitylene was used as an internal reference.

\subsection{Hydrosilylation of 1-hexene}

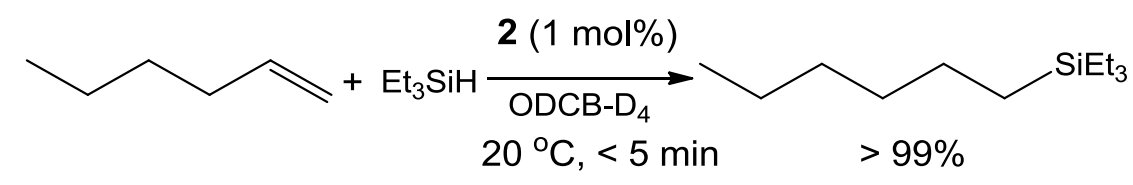

Scheme S1: Catalytic hydrosilylation of 1-hexene.

$1 \mathrm{mmol} \mathrm{Et}_{3} \mathrm{SiH}+1 \mathrm{mmol} 1-$ Hexene $+0.001 \mathrm{mmol}$ of compound 2
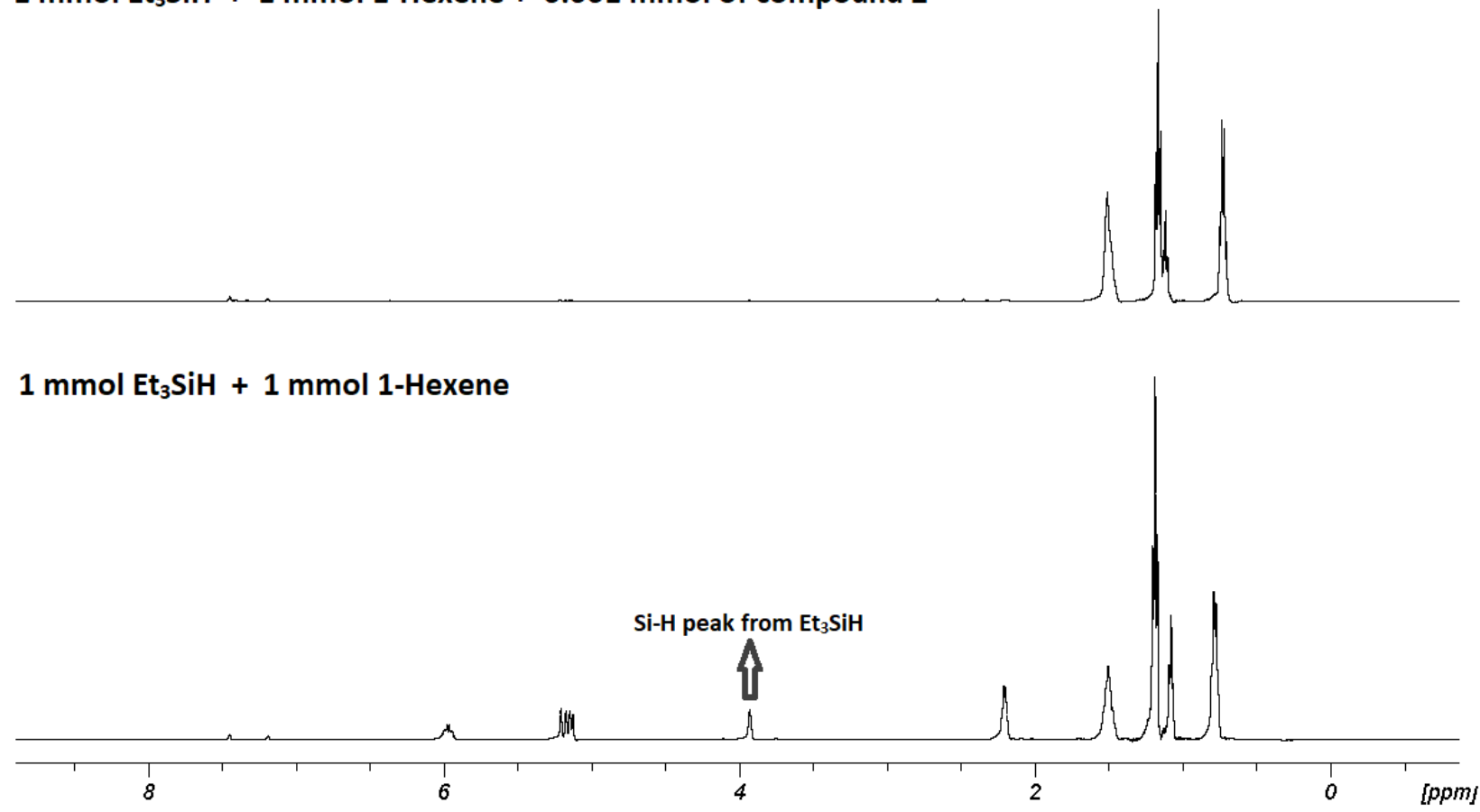

Figure S11: Stacking of ${ }^{1} \mathrm{H}$ NMR spectra of hydrosilylation mixture $\left(\mathrm{Et}_{3} \mathrm{SiH}\right.$ and 1-hexene) before (bottom) and after (top) the addition of compound 2 recorded in in ortho-dichlorobenzene-D4.

\subsubsection{Characterization of triethyl(hexyl)silane}

${ }^{1} \mathrm{H}$ NMR (ortho-dichlorobenzene- $\mathrm{D}_{4}, 500 \mathrm{MHz}$ ) : $\delta: 1.23\left(\mathrm{~m}, \mathrm{CH}_{2} \mathrm{CH}_{2} \mathrm{CH}_{2} \mathrm{CH}_{2}, 8 \mathrm{H}\right.$ ), $\delta 0.89$ (t, $\left.\mathrm{SiCH}_{2} \mathrm{CH}_{3}, 9 \mathrm{H}\right)$, $\delta 0.84$ (t, $\left.-\mathrm{CH}_{2} \mathrm{CH}_{3}, 3 \mathrm{H}\right), \delta 0.45\left(\mathrm{~m}, \mathrm{SiCH}_{2}, 8 \mathrm{H}\right)$.

${ }^{13} \mathrm{C}$ NMR (ortho-dichlorobenzene- $\left.\mathrm{D}_{4}, 125 \mathrm{MHz}\right): \delta 34.0\left(\mathrm{CH}_{2}\right), \delta 31.9\left(\mathrm{CH}_{2}\right), \delta 24.2\left(\mathrm{CH}_{2}\right), \delta 23.0$ $\left(\mathrm{CH}_{2}\right), \delta 14.4\left(\mathrm{CH}_{2} \mathrm{CH}_{3}\right), \delta 11.16\left(\mathrm{SiCH}_{2}\right), \delta 7.7\left(\mathrm{SiCH}_{2} \mathrm{CH}_{3}\right), \delta 3.7\left(\mathrm{SiCH}_{2}\right)$. 


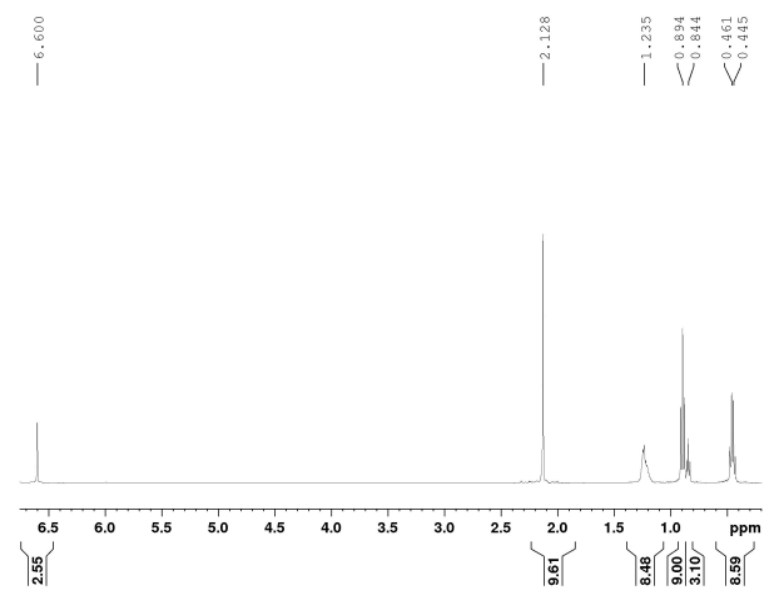

Figure S12: ${ }^{1} \mathrm{H}$ NMR spectrum of triethyl(hexyl)silane in ortho-dichlorobenzene-D4.

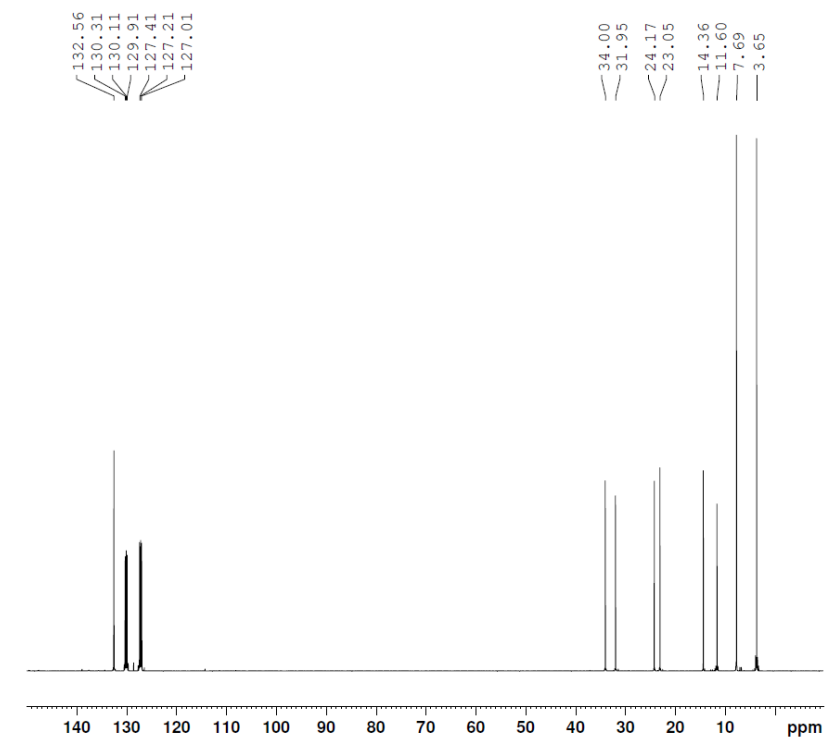

Figure S13: ${ }^{13} \mathrm{C}$ NMR spectrum of triethyl(hexyl)silane in ortho-dichlorobenzene-D4. 


\subsection{Hydrosilylation of Cyclohexene}
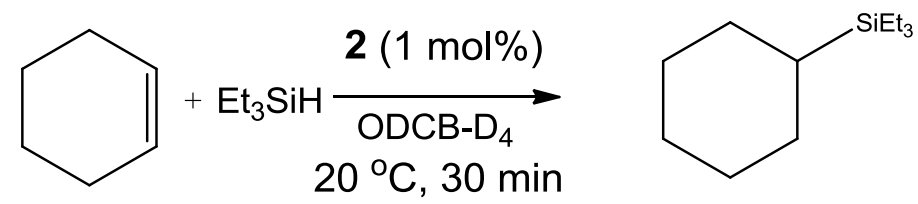

$>99 \%$

Scheme S2: Catalytic hydrosilylation of cyclohexene.

$1 \mathrm{mmol} \mathrm{Et} 3 \mathrm{SiH}+1 \mathrm{mmol}$ Cyclohexene $+0.001 \mathrm{mmol}$ of compound 2

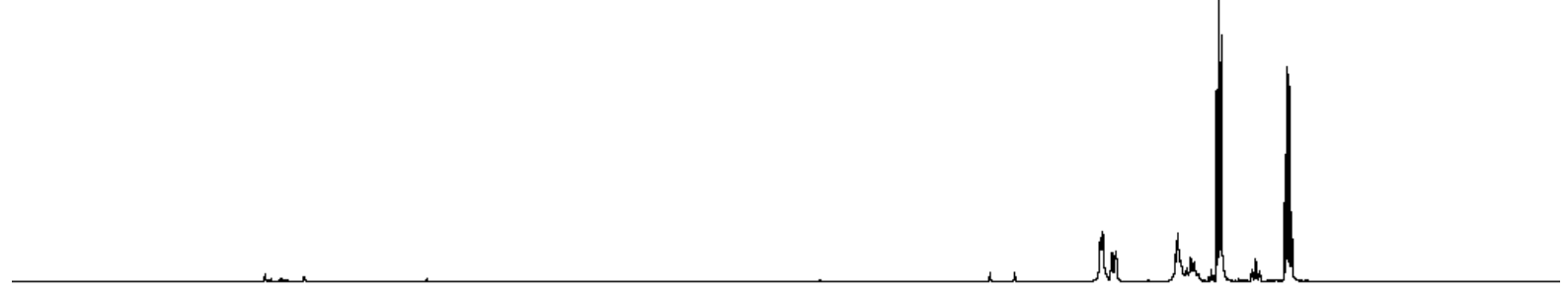

$1 \mathrm{mmol} \mathrm{Et}_{3} \mathrm{SiH}+1 \mathrm{mmol}$ Cyclohexene

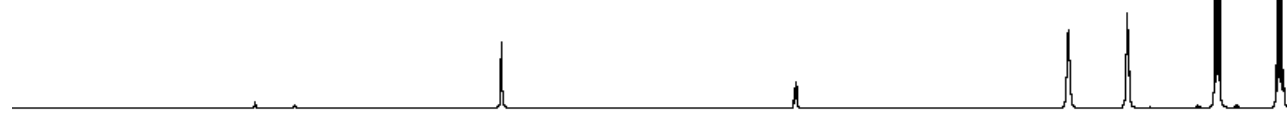

$8+1,19$

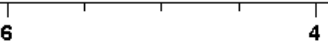

ppm

Figure S14: Stacking of ${ }^{1} \mathrm{H}$ NMR spectra of hydrosilylation mixture ( $\mathrm{Et}_{3} \mathrm{SiH}$ and $\left.\mathrm{Cyclohexene}\right)$ before (bottom) and after (top) the addition of compound 2 in ortho-dichlorobenzene-D4.

\subsubsection{Characterization of triethyl(cyclohexyl)silane}

${ }^{1} \mathrm{H}$ NMR (ortho-dichlorobenzene- $\left.\mathrm{D}_{4}, 500 \mathrm{MHz}\right): \delta: 1.92\left(\mathrm{~m}, \mathrm{CH}_{2} \mathrm{CH}_{2} \mathrm{CH}(\mathrm{H}), 5 \mathrm{H}\right), \delta: 1.42(\mathrm{~m}$, $\left.\mathrm{CH}_{2} \mathrm{CH}_{2} \mathrm{CH}(\mathrm{H}), 5 \mathrm{H}\right), \delta 1.14\left(\mathrm{t}, \mathrm{SiCH}_{2} \mathrm{CH}_{3}, 9 \mathrm{H}\right), \delta 0.90\left(\mathrm{~m},-\mathrm{SiCHCH}_{3}, 1 \mathrm{H}\right), \delta 0.69(\mathrm{~m}, \mathrm{SiCH}, 6 \mathrm{H})$.

${ }^{13} \mathrm{C}$ NMR (ortho-dichlorobenzene- $\left.\mathrm{D}_{4}, 125 \mathrm{MHz}\right): \delta 28.6\left(\mathrm{CH}_{2}\right), \delta 28.1\left(\mathrm{CH}_{2}\right)$, . $\delta 27.1\left(\mathrm{CH}_{2}\right), \delta 23.8$ $(\mathrm{SiCH}), \delta 7.9\left(\mathrm{SiCH}_{2} \mathrm{CH}_{3}\right), \delta 2.2(\mathrm{SiCH})$. 


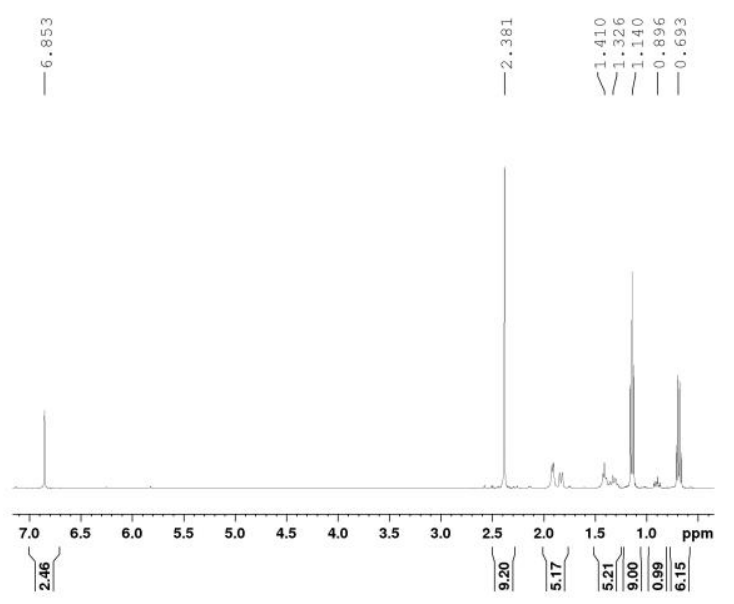

Figure S15: ${ }^{1} \mathrm{H}$ NMR spectrum of triethyl(cyclohexyl)silane in ortho-dichlorobenzene-D4.

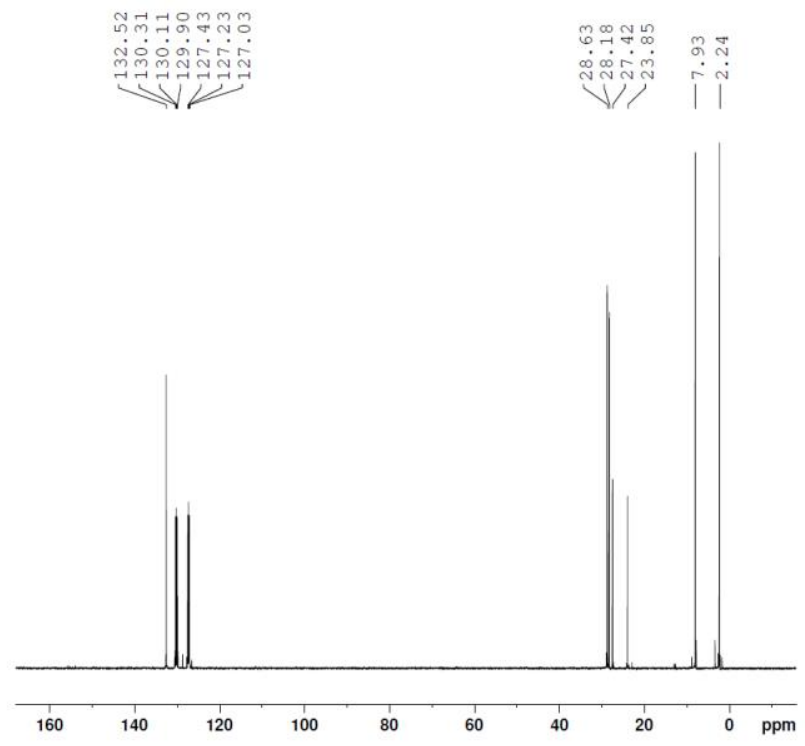

Figure S16: ${ }^{13} \mathrm{C}$ NMR spectrum of triethyl(cyclohexyl)silane in ortho-dichlorobenzene-D4. 


\subsection{Hydrosilylation of Styrene}

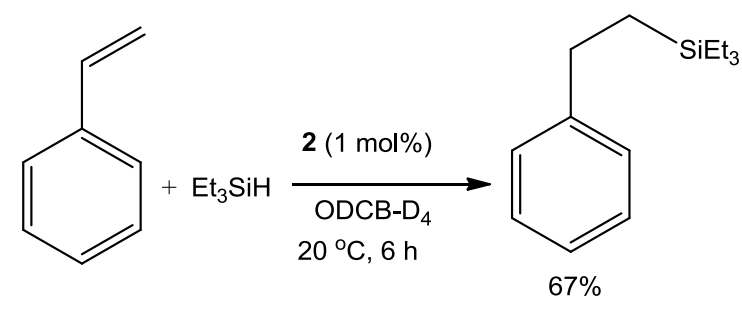

Scheme S3: Catalytic hydrosilylation of styrene.

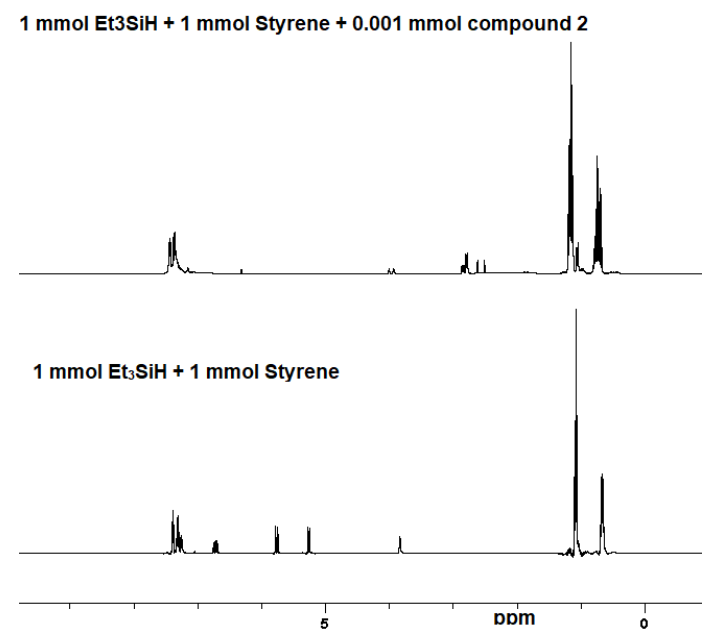

Figure S17: Stacking of ${ }^{1} \mathrm{H}$ NMR spectra of hydrosilylation mixture ( $\mathrm{Et}_{3} \mathrm{SiH}$ and styrene) before (bottom) and after (top) the addition of compound 2 in ortho-dichlorobenzene-D4.

\subsubsection{Characterization of triethyl(phenylethyl)silane}

${ }^{1} \mathrm{H}$ NMR (ortho-dichlorobenzene- $\left.\mathrm{D}_{4}, 500 \mathrm{MHz}\right): \delta 7.37\left(\mathrm{~m}, \mathrm{C}_{6} \mathrm{H}_{5}, 5 \mathrm{H}\right), \delta 2.81\left(\mathrm{~m}, \mathrm{SiCH}_{2} \mathrm{CH}, 2 \mathrm{H}\right), . \delta$ $1.16\left(\mathrm{~m}, \mathrm{SiCH}_{2} \mathrm{CH}_{3}, 9 \mathrm{H}\right), \delta 1.04\left(\mathrm{~m}, \mathrm{SiCH} \mathrm{CH}_{2}, 2 \mathrm{H}\right), \delta 0.75\left(\mathrm{~m}, \mathrm{SiCH}_{2} \mathrm{CH}_{3}, 6 \mathrm{H}\right)$.

${ }^{13} \mathrm{C}$ NMR (ortho-dichlorobenzene- $\left.\left.\mathrm{D}_{4}, 125 \mathrm{MHz}\right): \delta 128.5, \mathrm{C}_{6} \mathrm{H}_{5}\right), \delta 30.35\left(\mathrm{C}_{6} \mathrm{H}_{5} \mathrm{CH}_{2}\right), \delta 7.68$ $\left(\mathrm{SiCH}_{2} \mathrm{CH}_{3}\right)$, $\delta 3.05\left(\mathrm{SiCH} \mathrm{CH}_{2}\right)$, $\delta 2.76\left(\mathrm{SiCH}_{2} \mathrm{CH}_{3}\right)$. 


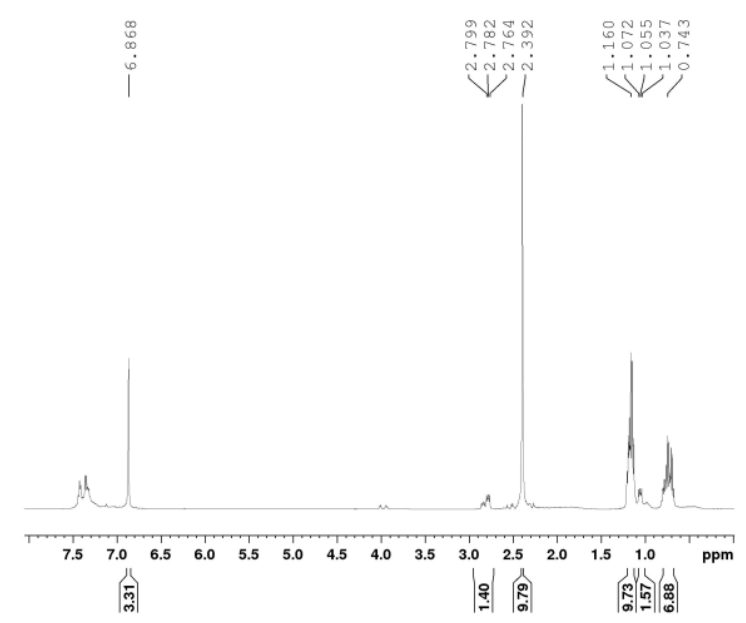

Figure S18: ${ }^{1} \mathrm{H}$ NMR spectrum of triethyl(phenylethyl)silane in ortho-dichlorobenzene-D4.

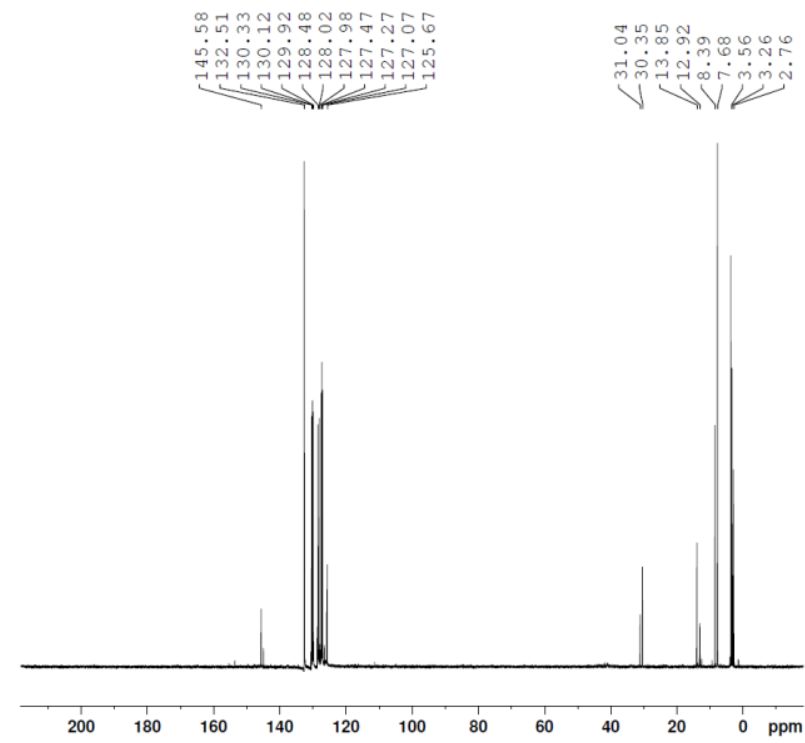

Figure S19: ${ }^{13} \mathrm{C}$ NMR spectrum of triethyl(phenylethyl)silane in ortho-dichlorobenzene-D4. 


\subsection{Hydrosilylation of diphenylethylene}

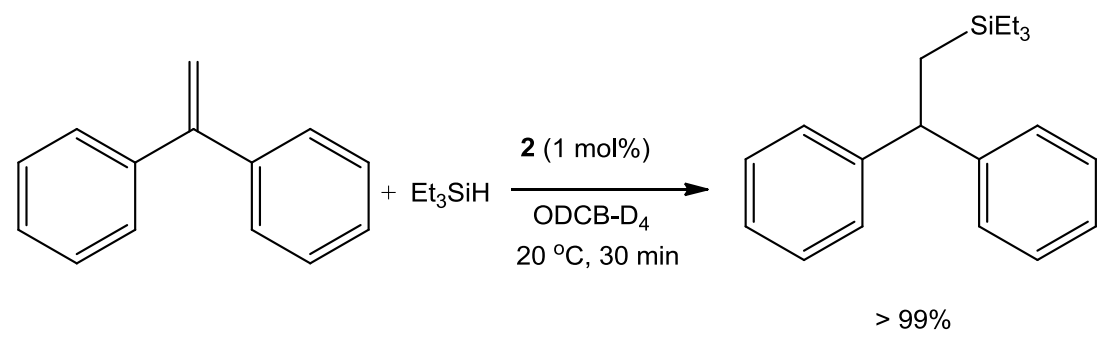

Scheme S4: Catalytic hydrosilylation of diphenylethylene.

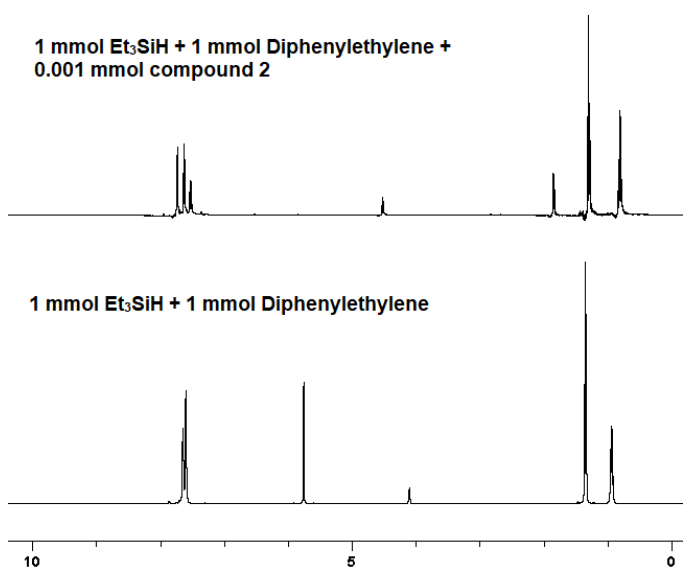

Figure S20: Stacking of ${ }^{1} \mathrm{H}$ NMR spectra of hydrosilylation mixture ( $\mathrm{Et}_{3} \mathrm{SiH}$ and diphenylethylene) before (bottom) and after (top) the addition of compound 2 in ortho-dichlorobenzene-D4.

\subsubsection{Characterization of (2,2-diphenylethyl)triethylsilane}

${ }^{1} \mathrm{H}$ NMR (ortho-dichlorobenzene- $\mathrm{D}_{4}, 500 \mathrm{MHz}$ ) : $\delta 7.56$ (d, o-CH-Phenyl, 4H), $\delta 7.45$ (t, m-CHPhenyl, 4H), $\delta 7.35$ (t, p-CH-Phenyl, 2H), $\delta 4.34$ (t, $\left.\mathrm{Ph}_{2} \mathrm{CH}, 1 \mathrm{H}\right), \delta 2.05$ (d, $\left.\mathrm{CH}_{2} \mathrm{CH}, 2 \mathrm{H}\right), \delta 1.12$ (t, $\mathrm{SiCH}_{2} \mathrm{CH}_{3}, 9 \mathrm{H}$ ), $\delta 0.63$ (q, $\mathrm{SiCH}_{2} \mathrm{CH}_{3}, 6 \mathrm{H}$ ).

${ }^{13} \mathrm{C}$ NMR (ortho-dichlorobenzene- $\left.\mathrm{D}_{4}, 125 \mathrm{MHz}\right): \delta 127.7\left(\mathrm{C}_{6} \mathrm{H}_{5}\right), \delta 47.58\left(\mathrm{C}_{6} \mathrm{H}_{5} \mathrm{CH}\right), \delta 19.30$ $\left(\mathrm{SiCH} \mathrm{H}_{2} \mathrm{CH}\right), \delta 7.68\left(\mathrm{SiCH}_{2} \mathrm{CH}_{3}\right), \delta 3.89\left(\mathrm{SiCH}_{2} \mathrm{CH}_{3}\right)$. 


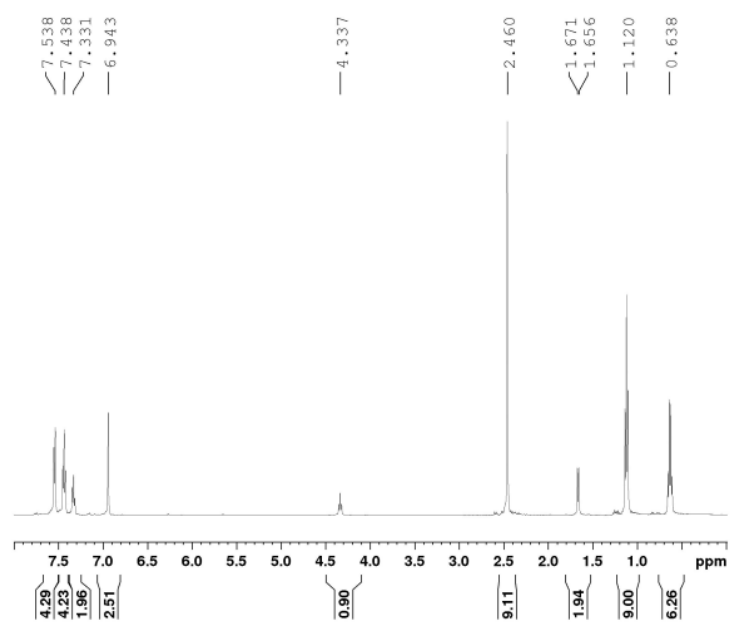

Figure S21: ${ }^{1} \mathrm{H}$ NMR spectrum of (2,2-diphenylethyl)triethylsilane in ortho-dichlorobenzene-D4.

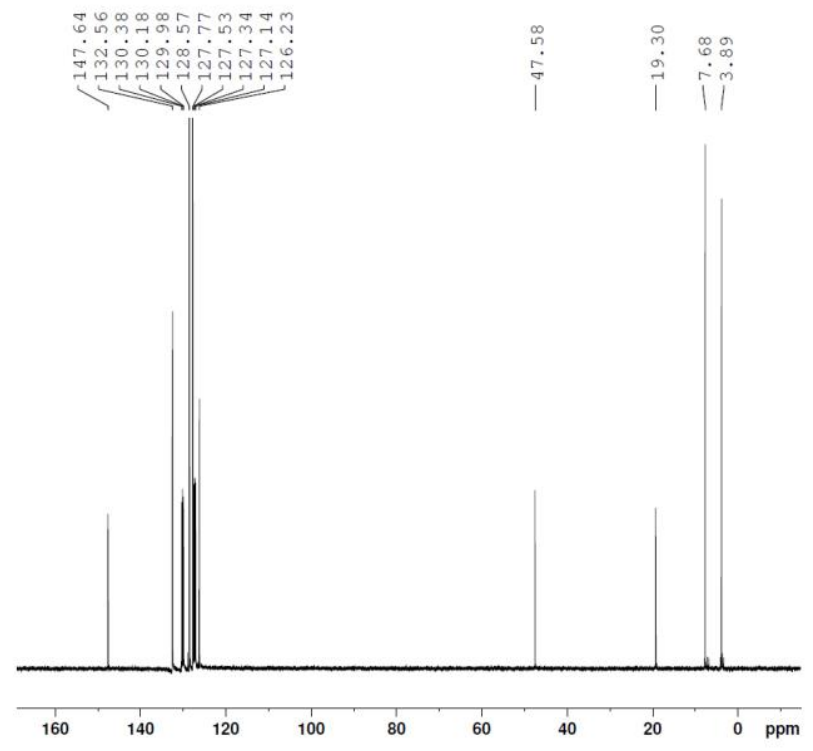

Figure S22: ${ }^{13} \mathrm{C}$ NMR spectrum of (2,2-diphenylethyl)triethylsilane in ortho-dichlorobenzene-D4. 


\subsection{Hydrosilylation of diphenylacetylene}

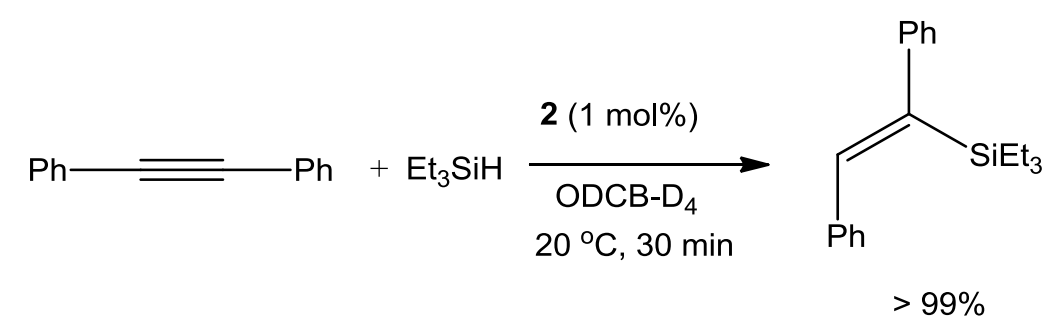

Scheme S5: Catalytic hydrosilylation of diphenylacetylene.

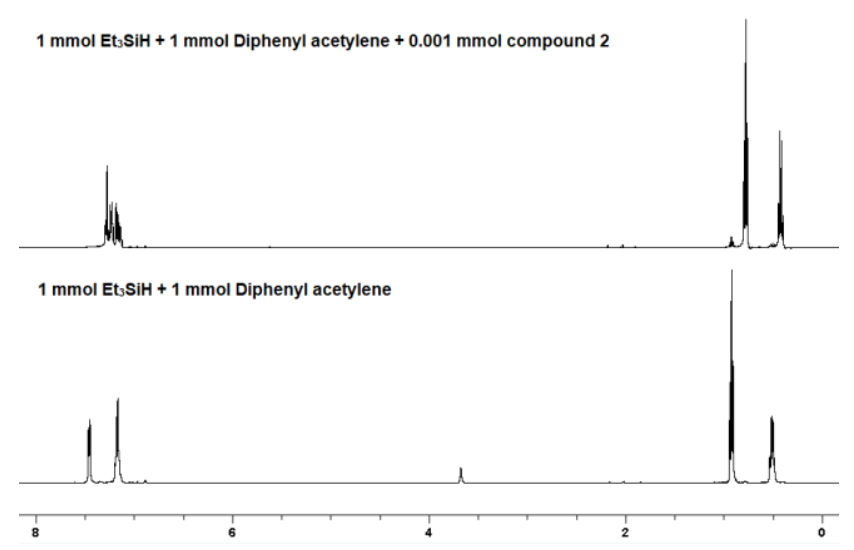

Figure S23: Stacking of ${ }^{1} \mathrm{H}$ spectra of hydrosilylation mixture ( $\mathrm{Et}_{3} \mathrm{SiH}$ and diphenylacetylene) before (bottom) and after (top) the addition of compound $\mathbf{2}$ in ortho-dichlorobenzene-D4.

\subsubsection{Characterization of (2,2-diphenylvinyl)triethylsilane}

${ }^{1} \mathrm{H}$ NMR (ortho-dichlorobenzene- $\left.\mathrm{D}_{4}, 500 \mathrm{MHz}\right): \delta 7.2\left(\mathrm{~m}, \mathrm{C}_{6} \mathrm{H}_{5}, 10 \mathrm{H}\right), \delta 7.2(\mathrm{~m}, \mathrm{CH}, 1 \mathrm{H}), \delta 0.78(\mathrm{~m}$, $\left.\mathrm{SiCH}_{2} \mathrm{CH}_{3}, 9 \mathrm{H}\right)$, $\delta 0.43$ (m, $\left.\mathrm{SiCH} \mathrm{CH}_{3}, 6 \mathrm{H}\right)$.

${ }^{13} \mathrm{C}$ NMR (ortho-dichlorobenzene- $\left.\mathrm{D}_{4}, 125 \mathrm{MHz}\right): \delta 146.9(\mathrm{CH}), \delta 139.9\left(\mathrm{C}_{6} \mathrm{H}_{5} \mathrm{C}\right), \delta 127.3\left(\mathrm{C}_{6} \mathrm{H}_{5}\right), \delta$ $7.58\left(\mathrm{SiCH}_{2} \mathrm{CH}_{3}\right), \delta 4.58\left(\mathrm{SiCH}_{2} \mathrm{CH}_{3}\right)$. 


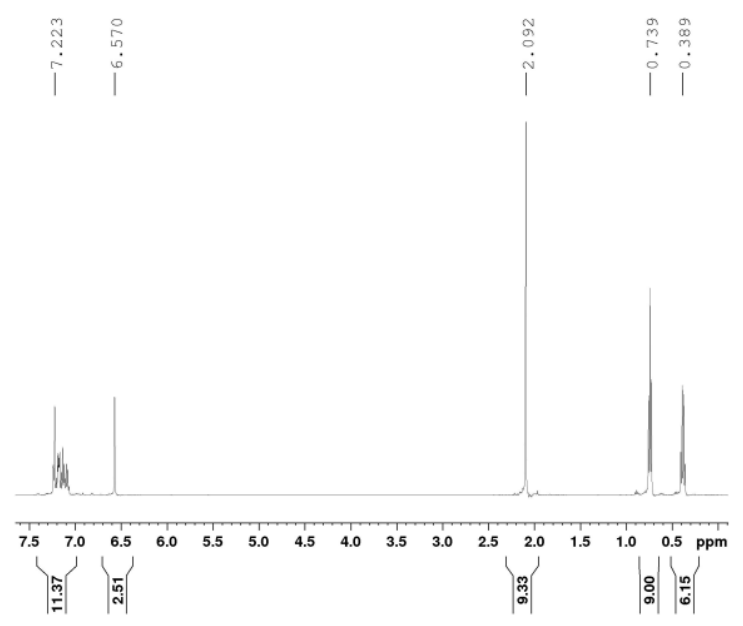

Figure S24: ${ }^{1} \mathrm{H}$ NMR spectrum of (2,2-diphenylvinyl)triethylsilane in ortho-dichlorobenzene-D4.

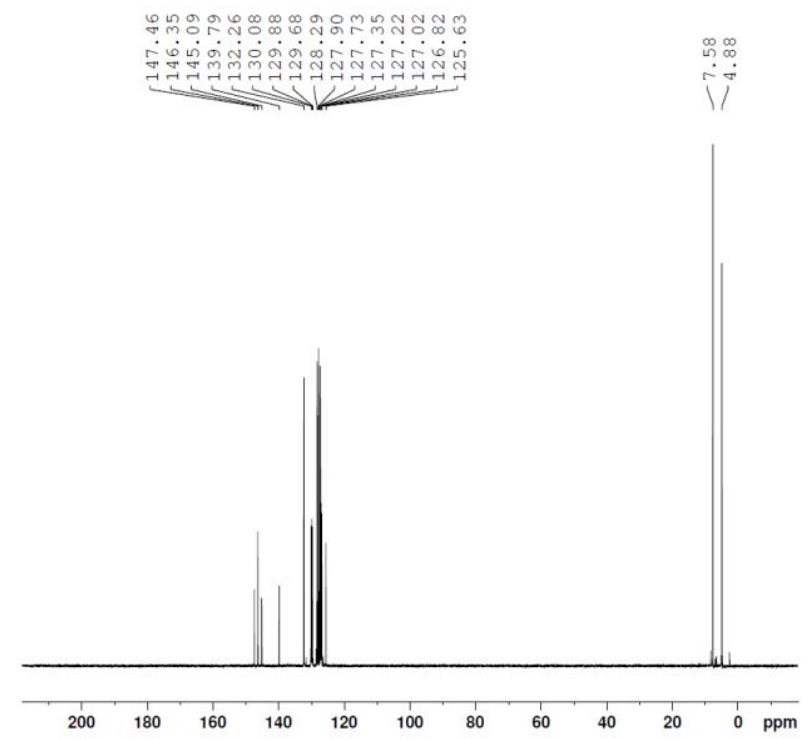

Figure S25: ${ }^{13} \mathrm{C}$ NMR spectrum of (2,2-diphenylvinyl)triethylsilane in ortho-dichlorobenzene-D4. 


\section{Crystallographic Details:}

Single-crystal X-ray crystallography for structural analysis was performed with a Bruker Kappa ApexII CCD diffractometer at $298 \mathrm{~K}$ and with Mo-Ka irradiation $(\lambda=0.71073 \AA)$. The structures were solved by direct methods using either SHELX2014 or SHELX-2017. Crystallographic data, details of data collection and structure refinement parameters for compounds $\mathbf{1}$, and $\mathbf{2}$ are presented below:

Table S1. Crystal data and structure refinement for 1 .

Identification code

CCDC Number

Empirical formula

Formula weight

Temperature

Wavelength

Crystal system

Space group

Unit cell dimensions

Volume

Z

Density (calculated)

Absorption coefficient

$\mathrm{F}(000)$

Crystal size

Theta range for data collection

Index ranges

Reflections collected

Independent reflections

Completeness to theta $=26.000^{\circ}$

Absorption correction

Max. and min. transmission

Refinement method

Data / restraints / parameters

Goodness-of-fit on $\mathrm{F}^{2}$

Final $R$ indices [ $>2$ sigma( $(\mathrm{l})]$

$R$ indices (all data)

Extinction coefficient

Largest diff. peak and hole compound 1

CCDC 1410594

C15 H22 B Bi Cl2 N6

577.07

$150 \mathrm{~K}$

$0.71073 \AA$

Monoclinic

P 21/n

$a=14.6366(17) \AA \quad \alpha=90^{\circ}$.

$\mathrm{b}=7.7961(10) \AA \quad \beta=106.437(5)^{\circ}$.

$\mathrm{c}=17.985(2) \AA \quad \gamma=90^{\circ}$.

1968.4(4) $\AA^{3}$

4

$1.947 \mathrm{Mg} / \mathrm{m}^{3}$

$9.239 \mathrm{~mm}^{-1}$

1104

$0.150 \times 0.100 \times 0.100 \mathrm{~mm}^{3}$

2.867 to $25.996^{\circ}$.

$-17<=\mathrm{h}<=17,-9<=\mathrm{k}<=9,0<=1<=22$

6964

$6964[\mathrm{R}(\mathrm{int})=$ ?]

$97.0 \%$

Semi-empirical from equivalents

0.591 and 0.442

Full-matrix least-squares on $\mathrm{F}^{2}$

6964 / 172 / 236

1.073

$\mathrm{R} 1=0.0387, \mathrm{wR} 2=0.0993$

$\mathrm{R} 1=0.0496, \mathrm{wR} 2=0.1054$

$\mathrm{n} / \mathrm{a}$

1.239 and -2.217 e. $\AA^{-3}$ 
Table S2.Crystal data and structure refinement for 2.

Identification code

CCDC Number

Empirical formula

Formula weight

Temperature

Wavelength

Crystal system

Space group

Unit cell dimensions

Volume

Z

Density (calculated)

Absorption coefficient

$\mathrm{F}(000)$

Crystal size

Theta range for data collection

Index ranges

Reflections collected

Independent reflections

Completeness to theta $=25.000^{\circ}$

Absorption correction

Max. and min. transmission

Refinement method

Data / restraints / parameters

Goodness-of-fit on $\mathrm{F}^{2}$

Final $\mathrm{R}$ indices [l>2sigma(I)]

$R$ indices (all data)

Extinction coefficient

Largest diff. peak and hole compound2

CCDC 1847457

C93 H45.83 B3 Bi Cl6.18 F40 N6

2467.49

$150 \mathrm{~K}$

$0.71073 \AA$

Triclinic

P-1

$\mathrm{a}=13.48(5) \AA$

$\alpha=70.79(7)^{\circ}$.

$\mathrm{b}=16.02(6) \AA$

$\beta=80.64(7)^{\circ}$.

$\mathrm{c}=24.09(9) \AA$

$\gamma=67.07(6)^{\circ}$.

4523(29) $\AA^{3}$

2

$1.812 \mathrm{Mg} / \mathrm{m}^{3}$

$2.268 \mathrm{~mm}^{-1}$

2418

$0.150 \times 0.120 \times 0.012 \mathrm{~mm}^{3}$

2.547 to $25.000^{\circ}$.

$-16<=\mathrm{h}<=16,-19<=\mathrm{k}<=19,-28<=\mathrm{k}<=28$

41181

$15828[R($ int $)=0.1052]$

$99.5 \%$

Semi-empirical from equivalents

0.74 and 0.55

Full-matrix least-squares on $\mathrm{F}^{2}$

15828 / 430 / 1426

1.025

$\mathrm{R} 1=0.0698, \mathrm{wR} 2=0.1513$

$\mathrm{R} 1=0.1206, \mathrm{wR} 2=0.1786$

$0.0039(3)$

3.180 and -1.196 e. $\AA^{-3}$ 


\section{Computational Details:}

Quantum chemical calculations were performed using Gaussian $09^{[5]}$ quantum chemistry program package. All geometries were optimized using density functional theory (DFT) with the hybrid functional $\mathrm{PBEO}^{[6]}$ and def2-TZVP ${ }^{[7]}$ basis set for all atoms. The nature of stationary points were characterized by the Hessian matrix of force constants. All structures were obtained as minima on their potential energy surfaces (PES). Natural bond orbital (NBO ${ }^{[8]}$ analysis was carried out to analyse the nature of lone-pair and charge on the $\mathrm{Bi}$ atom.

The nature of Bi-N bonds was analyzed using energy partitioning scheme ${ }^{[9]}$ of Amsterdam Density Functional $(A D F)^{[10]}$ program package with the hybrid functional PBE0. A triple zeta plus double polarization (TZ2P) basis set was employed for all the atoms. Slater-type orbitals (STOs) were used as basis functions ${ }^{[11]}$. Scaler relativistic effects were considered using zero-order regular approximation (ZORA) $)^{[12]}$.

The interaction energy, $\Delta \mathrm{E}_{\text {int }}$ was calculated between $\mathrm{Bi}^{3+}$ and [Tp $\left.{ }^{\mathrm{Me}}\right]^{-}$fragments for the complex 2 and $\mathrm{BiCl}_{2}{ }^{+}$and $\left[\mathrm{Tp}^{\mathrm{Me}}{ }^{\mathrm{e}}\right]$ for the complex 1. Further, the total interaction energy, $\Delta \mathrm{E}_{\text {int }}$ was decomposed into three components:

$$
\Delta \mathrm{E}_{\text {int }}=\Delta \mathrm{E}_{\text {elstat }}+\Delta \mathrm{E}_{\text {Pauli }}+\Delta \mathrm{E}_{\text {orb }}
$$

The electrostatic $\left(\Delta \mathrm{E}_{\text {elstat }}\right)$ interaction energy between two fragments is calculated using the frozen electron density distribution of the fragments in the complex. The repulsive $\left(\Delta \mathrm{E}_{\text {Pauli }}\right)$ interactions are calculated by enforcing the Kohn-Sham determinant on the superimposed fragments to obey the Pauli principle by anti-symmetrization and renormalization. The orbital interaction term $\left(\Delta \mathrm{E}_{\text {orb }}\right)$ constitutes the covalent contribution to the total interaction energy. This term accounts for all contributions resulting from electron pair bonding, charge transfer and intrafragment polarization. The topology of the electron density at bond critical point (BCP) was calculated using Quantum Theory of Atoms in Molecules (QTAIM) ${ }^{[13]}$. AIMALL ${ }^{[14]}$ program package was used with the wave function files generated at PBE0/Def2-TZVP method. 

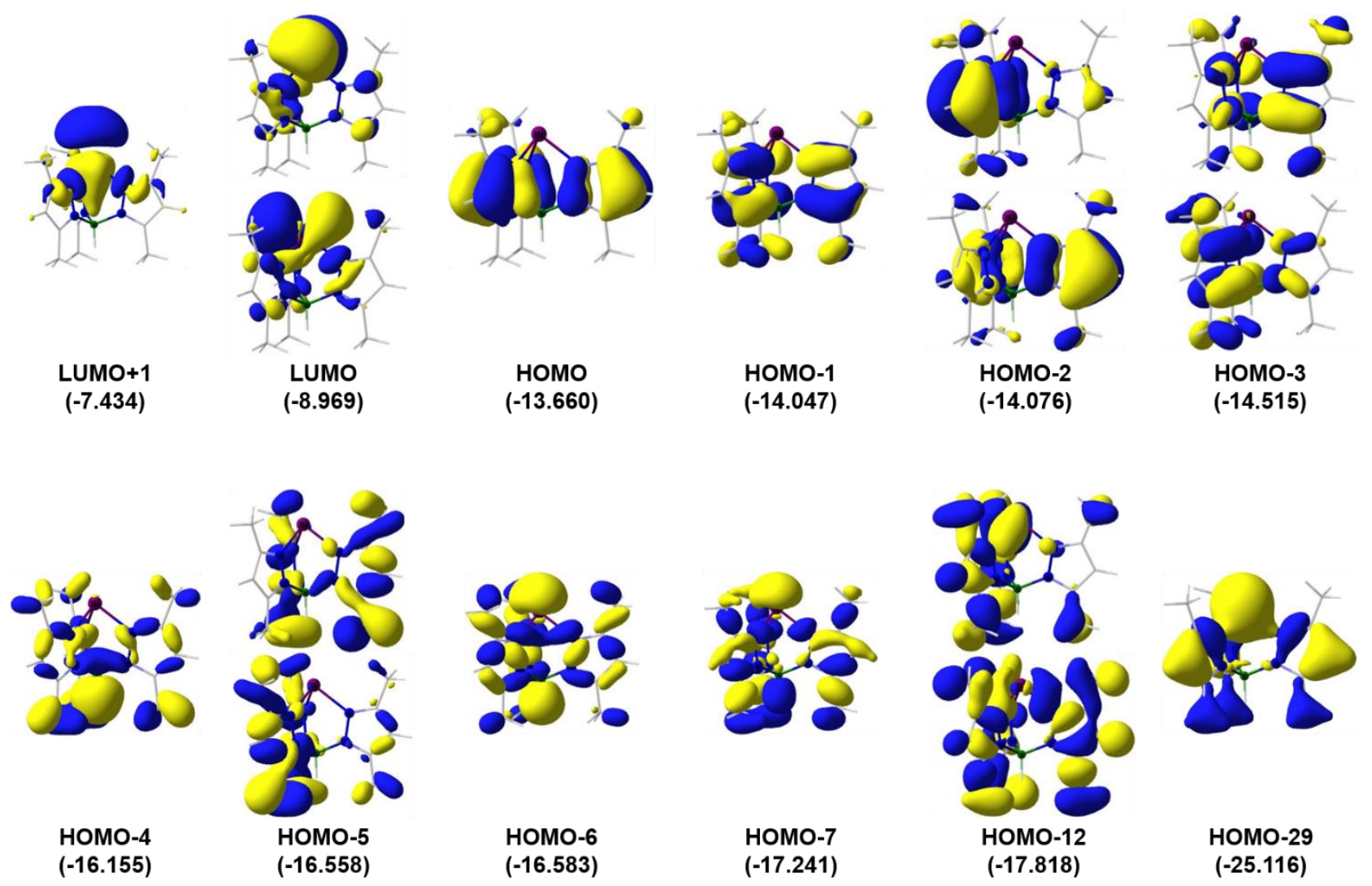

Figure S26. Plots of the frontier valence orbitals (isovalue $=0.03$ ) of $\left[\mathrm{Tp}^{\mathrm{Me}} \mathrm{Bi}^{2+}\right.$ molecule at PBE0 functional using def2-TZVP basis set for all atoms. The eigenvalues [eV] are given below.

The MO delocalized over three pyrazole rings of the ligand viz. HOMO, HOMO-1, HOMO-2, HOMO3, HOMO-4, HOMO-5, do not contribute to Bi-N bonding. So, these molecular orbitals are not considered in $\mathrm{MO}$ interaction diagram for simplicity (Fig 3). This makes HOMO-6 as the highest occupied orbital in the interaction diagram.

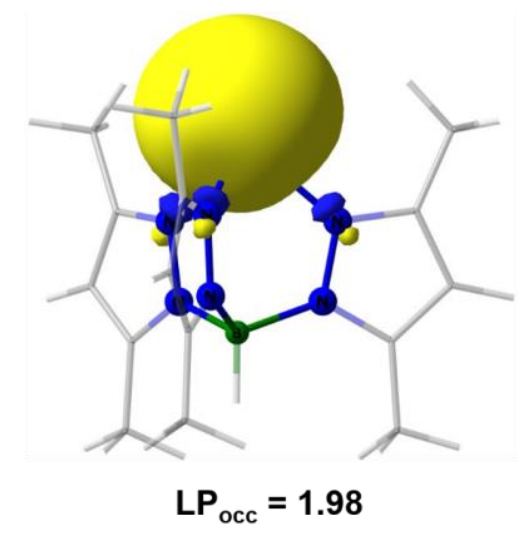

$(88.34 \% \mathrm{~s}, 11.64 \% \mathrm{p}, 0.00 \% \mathrm{~d})$

Figure S27. Plot of Bi centred NBO non-bonding lone-pair orbital of s-character (isovalue $=0.02$ ) for complex 2. 


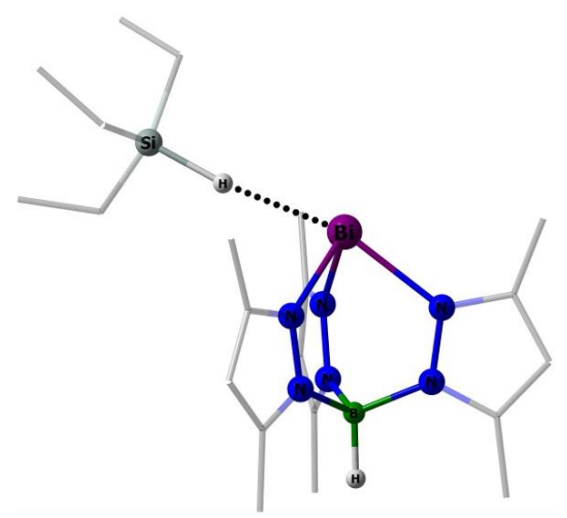

(a)

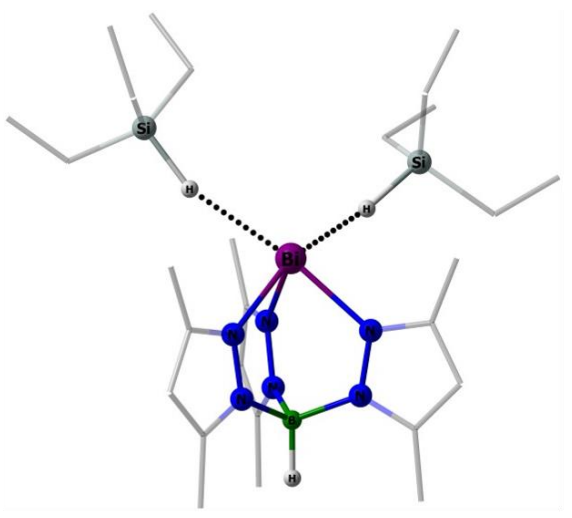

(b)

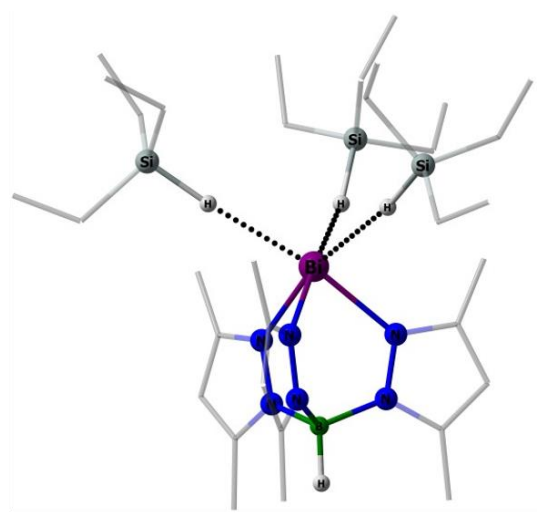

(c)

Figure S28. Optimized geometries $\left[\mathrm{Tp}^{\mathrm{Me} 2} \mathrm{Bi} \cdots\left(\mathrm{H}-\mathrm{SiEt}_{3}\right)_{n}\right]^{2+}(\mathrm{n}=1-3)$ complexes at DFT method using PBE0 functional with def2-TZVP basis set for all atoms.

Table S3. Selected interatomic distances $(\AA)$ and WBI index of $\left[\mathrm{Tp}^{\mathrm{Me} 2} \mathrm{Bi} \cdots\left(\mathrm{H}-\mathrm{SiEt}_{3}\right)_{n}\right]^{2+}(\mathrm{n}=1$ and 3$)$ calculated at PBE0 level with Def2-TZVP basis set for all atoms.

\begin{tabular}{cccc}
\hline & & Bond Length $(\AA)$ & WBI Index \\
\hline$\left.[\mathrm{Tp}-\mathrm{Bi}---\mathrm{H}-\mathrm{SiEt}]_{3}\right]^{2+}$ & $\mathrm{Bi}---\mathrm{H}$ & 2.394 & 0.13 \\
& $\mathrm{Si}-\mathrm{H}$ & 1.579 & 0.70 \\
{$\left[\mathrm{Tp}-\mathrm{Bi}---\left(\mathrm{H}-\mathrm{SiEt}_{3}\right)_{3}\right]^{2+}$} & $\mathrm{Bi}---\mathrm{H} 1$ & 2.555 & 0.106 \\
& $\mathrm{Bi}---\mathrm{H} 2$ & 2.601 & 0.097 \\
& $\mathrm{Bi}---\mathrm{H} 3$ & 2.622 & 0.094 \\
$\mathrm{Si} 1-\mathrm{H} 1$ & 1.556 & 0.75 \\
& $\mathrm{Si} 2-\mathrm{H} 2$ & 1.552 & 0.76 \\
& $\mathrm{Si} 3-\mathrm{H} 3$ & 1.550 & 0.77 \\
\hline
\end{tabular}


Table S4. Selected bond angles $\left(^{\circ}\right)$ and NBO charges of $\left[\mathrm{Tp}^{\mathrm{Me} 2} \mathrm{Bi} \cdots\left(\mathrm{H}-\mathrm{SiEt}_{3}\right)_{n}\right]^{2+}(\mathrm{n}=1-3)$ calculated at PBE0 level with Def2-TZVP basis set for all atoms.

\begin{tabular}{|c|c|c|c|}
\hline & & Bond Angle $\left({ }^{\circ}\right)$ & NBO Charge \\
\hline \multirow[t]{2}{*}[\mathrm{Tp}-\mathrm{Bi}---\mathrm{H}-\mathrm{SiEt}3]{$^{2+}$} & $\mathrm{Bi}-\mathrm{H}-\mathrm{Si}$ & 171.1 & $\mathrm{Si}+1.57$ \\
\hline & & & $\mathrm{H}-0.34$ \\
\hline \multirow[t]{4}{*}[\mathrm{Tp}-\mathrm{Bi}---(\mathrm{H}-\mathrm{SiEt}_{3})_{2}]{$^{2+}$} & Bi---H1-Si1 & 161.6 & Si1 +1.55 \\
\hline & & & $\mathrm{H} 1-0.32$ \\
\hline & Bi---H2-Si2 & 160.8 & $\mathrm{Si} 2+1.53$ \\
\hline & & & $\mathrm{H} 2-0.31$ \\
\hline \multirow[t]{6}{*}[\mathrm{Tp}-\mathrm{Bi}---(\mathrm{H}-\mathrm{SiEt}_{3})_{3}]{$^{2+}$} & Bi---H1-Si1 & 171.0 & Si1 +1.53 \\
\hline & & & H1 -0.31 \\
\hline & $\mathrm{Bi}---\mathrm{H} 2-\mathrm{Si} 2$ & 174.7 & Si2 +1.52 \\
\hline & & & $\mathrm{H} 2-0.31$ \\
\hline & Bi---H3-Si3 & 149.2 & Si3 +1.51 \\
\hline & & & H3 -0.29 \\
\hline
\end{tabular}
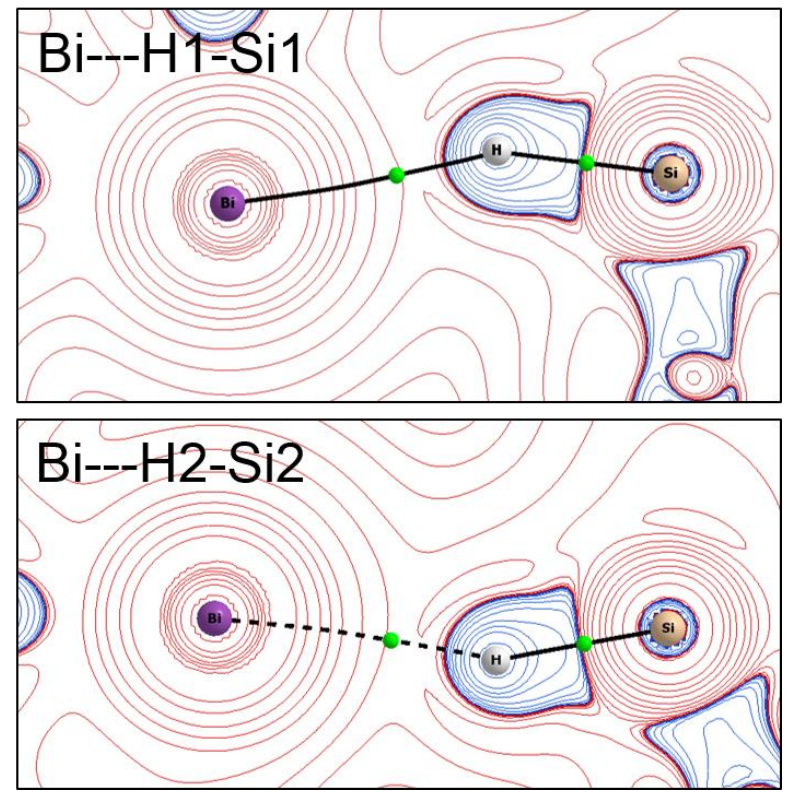

Figure S29. Contour line diagram of the Laplacian $-\nabla^{2} \rho(r)$ maps of $\sigma-\mathrm{Si} 1-\mathrm{H} 1-\mathrm{Bi}$ (Top) and $\sigma-\mathrm{Si} 2-\mathrm{H} 2-$ $\mathrm{Bi}$ (Down) complex in the plane defined by $\mathrm{Bi}, \mathrm{H} 1 / \mathrm{H} 2$ and $\mathrm{Si} 1 / \mathrm{Si} 2$ atoms. Bond paths are shown as black lines and BCP as solid green dots. 


\section{Optimized coordinates of molecules by DFT}

Cartesian coordinates and the total energy (a.u.) of the optimized structures using PBE0 hybrid functional and def2-TZVP basis set for all atoms.

Complex 1

$E_{\text {Total }}=\mathbf{- 2 0 7 2 . 4 2 2 6 0 3 8 1 ~ a . u . ~}$

$\begin{array}{llll}\text { H } & -3.13432600 & -0.02573100 & 1.05073000 \\ \mathrm{C} & 0.31837700 & -3.22901600 & -2.37493900 \\ \mathrm{H} & 0.12192300 & -4.27899800 & -2.59315300 \\ \mathrm{H} & 1.25838500 & -3.16242700 & -1.82398500 \\ \mathrm{H} & 0.44329900 & -2.71335200 & -3.33200700 \\ \mathrm{C} & -0.80555900 & -2.64715500 & -1.59378000 \\ \mathrm{C} & -2.12101900 & -3.10516300 & -1.48431400 \\ \mathrm{H} & -2.54687500 & -3.98495900 & -1.93982100 \\ \mathrm{C} & -2.76648100 & -2.20198000 & -0.66053300 \\ \mathrm{C} & -4.18061200 & -2.20450900 & -0.20347100 \\ \mathrm{H} & -4.25445800 & -2.23872200 & 0.88561900 \\ \mathrm{H} & -4.69044500 & -3.07955900 & -0.60604100 \\ \mathrm{H} & -4.71785900 & -1.31524900 & -0.54075600 \\ \mathrm{C} & 0.29775300 & 3.31201700 & -2.26546300 \\ \mathrm{H} & 0.09241500 & 4.36622700 & -2.45289700 \\ \mathrm{H} & 0.42839900 & 2.82586400 & -3.23712000 \\ \mathrm{H} & 1.23750800 & 3.23709500 & -1.71527000 \\ \mathrm{C} & -0.82225100 & 2.69754900 & -1.50393100 \\ \mathrm{C} & -2.14145000 & 3.14161500 & -1.38273700 \\ \mathrm{H} & -2.57331200 & 4.03199800 & -1.81122500 \\ \mathrm{C} & -2.78139400 & 2.20784800 & -0.58933900 \\ \mathrm{C} & -4.19643100 & 2.18504100 & -0.13562500 \\ \mathrm{H} & -4.71415800 & 3.06439300 & -0.51826400 \\ \mathrm{H} & -4.27312400 & 2.19266300 & 0.95383500 \\ \mathrm{H} & -4.72395500 & 1.29878000 & -0.49526800 \\ \mathrm{C} & 2.39906500 & -0.08504700 & 2.98053100 \\ \mathrm{H} & 2.90103000 & 0.73202300 & 2.46150100\end{array}$




$\begin{array}{lrrr}\mathrm{H} & 2.83774800 & -1.01729600 & 2.62156200 \\ \mathrm{H} & 2.58548900 & 0.00589500 & 4.05041700 \\ \mathrm{C} & 0.93540400 & -0.06115400 & 2.74255600 \\ \mathrm{C} & -0.06241000 & -0.07439400 & 3.71008100 \\ \mathrm{H} & 0.08059000 & -0.09498300 & 4.77817600 \\ \mathrm{C} & -1.26571000 & -0.05594800 & 3.03209500 \\ \mathrm{C} & -2.63588000 & -0.06074900 & 3.60706100 \\ \mathrm{H} & -3.20096400 & 0.82607700 & 3.31411800 \\ \mathrm{H} & -2.56586600 & -0.07728400 & 4.69447100 \\ \mathrm{H} & -3.20564400 & -0.93548500 & 3.28786100 \\ \mathrm{~N} & -0.67324800 & -1.53497000 & -0.87474200 \\ \mathrm{~N} & -1.86681000 & -1.26349400 & -0.30963100 \\ \mathrm{~N} & -0.68249800 & 1.56439600 & -0.81975800 \\ \mathrm{~N} & -1.87498500 & 1.26594400 & -0.26622100 \\ \mathrm{~N} & 0.34753400 & -0.03356800 & 1.53705900 \\ \mathrm{~N} & -1.00143800 & -0.03132100 & 1.71599100 \\ \mathrm{~B} & -2.04142100 & -0.01378300 & 0.56735900 \\ \mathrm{Bi} & 1.29558400 & 0.01517400 & -0.54672400 \\ \mathrm{Cl} & 2.79313200 & -1.95712400 & 0.12241200 \\ \mathrm{Cl} & 2.76683900 & 1.98476600 & 0.19292900\end{array}$

\section{Complex 2}

$$
\begin{array}{lrrr}
\multicolumn{4}{l}{\mathbf{E}_{\text {Total }}=\mathbf{- 1 1 5 1 . 6 6 1 4 2 5 9 4} \text { a.u. }} \\
\mathrm{H} & 2.93302400 & 0.00250400 & -0.00313000 \\
\mathrm{C} & -1.71811600 & 3.60138500 & -0.32193500 \\
\mathrm{H} & -1.71681400 & 4.61166600 & 0.08658100 \\
\mathrm{H} & -2.45309200 & 3.02594300 & 0.24864000 \\
\mathrm{H} & -2.06049300 & 3.67003900 & -1.35834500 \\
\mathrm{C} & -0.35686800 & 3.01719100 & -0.25023200 \\
\mathrm{C} & 0.87821600 & 3.63190100 & -0.31073100 \\
\mathrm{H} & 1.06599100 & 4.69019900 & -0.39859900
\end{array}
$$




$\begin{array}{llll}\mathrm{C} & 1.83949500 & 2.62613100 & -0.23385100 \\ \mathrm{C} & 3.31481500 & 2.76038800 & -0.25321400 \\ \mathrm{H} & 3.76236600 & 2.37051300 & 0.66307000 \\ \mathrm{H} & 3.58527500 & 3.81090900 & -0.34334100 \\ \mathrm{H} & 3.75240700 & 2.22237400 & -1.09639000 \\ \mathrm{C} & -1.71626600 & -2.08269300 & -2.95628200 \\ \mathrm{H} & -2.04666100 & -3.02762300 & -2.51595700 \\ \mathrm{H} & -1.72068000 & -2.21010700 & -4.03864600 \\ \mathrm{H} & -2.45753400 & -1.31325300 & -2.72103700 \\ \mathrm{C} & -0.35547700 & -1.72625300 & -2.48680800 \\ \mathrm{C} & 0.87990200 & -2.08459300 & -2.98933600 \\ \mathrm{H} & 1.06818300 & -2.69011900 & -3.86161400 \\ \mathrm{C} & 1.84077000 & -1.51241500 & -2.15827100 \\ \mathrm{C} & 3.31614800 & -1.59404500 & -2.26614200 \\ \mathrm{H} & 3.58665800 & -2.19356100 & -3.13347600 \\ \mathrm{H} & 3.75479500 & -2.05868400 & -1.38086900 \\ \mathrm{H} & 3.76272300 & -0.60473700 & -2.38281900 \\ \mathrm{C} & -1.70922600 & -1.52385300 & 3.28369200 \\ \mathrm{H} & -2.45685100 & -1.67888800 & 2.50021400 \\ \mathrm{H} & -2.02921100 & -0.68237000 & 3.90443200 \\ \mathrm{H} & -1.71699800 & -2.41333000 & 3.91355700 \\ \mathrm{C} & -0.34986700 & -1.29233100 & 2.73817200 \\ \mathrm{C} & 0.88677000 & -1.54675100 & 3.29799400 \\ \mathrm{H} & 1.07707300 & -1.99994900 & 4.25787500 \\ \mathrm{C} & 1.84557100 & -1.10974200 & 2.38657900 \\ \mathrm{C} & 3.32121700 & -1.15881100 & 2.50946800 \\ \mathrm{H} & 3.76831000 & -1.75343500 & 1.71050200 \\ \mathrm{H} & 3.59398400 & -1.60948800 & 3.46201500 \\ \mathrm{H} & 3.75733900 & -0.15876000 & 2.46853000 \\ \mathrm{~N} & -0.13844200 & 1.68820900 & -0.13804700 \\ \mathrm{~N} & 1.20353500 & 1.44868700 & -0.12933000 \\ \mathrm{~N} & -0.13750300 & -0.96322400 & -1.39287200 \\ \mathrm{~N} & 1.20434600 & -0.83313500 & -1.19114900 \\ \mathrm{~N} & -0.13448700 & -0.72518900 & 1.53069300\end{array}$




$\begin{array}{lrrr}\text { N } & 1.20688400 & -0.61183400 & 1.31596300 \\ \mathrm{~B} & 1.74526600 & 0.00166700 & -0.00202700 \\ \mathrm{Bi} & -1.48757800 & -0.00091400 & 0.00153600\end{array}$

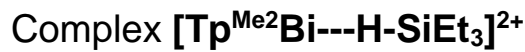

$E_{\text {TOTAL }}=-1679.14163606$ a.u.

$\begin{array}{llrr}\text { H } & 3.16442500 & -0.21285900 & 2.14650100 \\ \mathrm{C} & 2.76394800 & 0.16229200 & -3.70547600 \\ \mathrm{H} & 3.46583800 & 0.74063400 & -4.30613600 \\ \mathrm{H} & 1.78646000 & 0.64362800 & -3.79791000 \\ \mathrm{H} & 2.69850100 & -0.83416500 & -4.15058100 \\ \mathrm{C} & 3.22965900 & 0.08543800 & -2.29818800 \\ \mathrm{C} & 4.52050300 & 0.01930300 & -1.79992500 \\ \mathrm{H} & 5.43491400 & 0.03697200 & -2.37104400 \\ \mathrm{C} & 4.41123100 & -0.07183900 & -0.41748100 \\ \mathrm{C} & 5.49333600 & -0.16461500 & 0.59159900 \\ \mathrm{H} & 5.45939300 & 0.67153100 & 1.29263300 \\ \mathrm{H} & 6.46084900 & -0.14945800 & 0.09296300 \\ \mathrm{H} & 5.42348400 & -1.08889900 & 1.16846800 \\ \mathrm{C} & -1.16379800 & -3.18939800 & -0.47963800 \\ \mathrm{H} & -1.82345800 & -3.89021500 & 0.03133200 \\ \mathrm{H} & -0.71870100 & -3.72130800 & -1.32542900 \\ \mathrm{H} & -1.78360300 & -2.37737300 & -0.86749100 \\ \mathrm{C} & -0.11299300 & -2.70066400 & 0.44407200 \\ \mathrm{C} & 0.49512300 & -3.33974600 & 1.50763700 \\ \mathrm{H} & 0.26514700 & -4.32331900 & 1.88481700 \\ \mathrm{C} & 1.47046700 & -2.47444200 & 1.99416200 \\ \mathrm{C} & 2.41103000 & -2.68727200 & 3.11984800 \\ \mathrm{H} & 2.19280900 & -3.63786900 & 3.60339600 \\ \mathrm{H} & 2.32826400 & -1.89560800 & 3.86628800 \\ \mathrm{H} & 3.44591600 & -2.71385900 & 2.77250000\end{array}$




\begin{tabular}{|c|c|c|c|}
\hline C & -0.89272800 & 3.41099200 & -0.06409800 \\
\hline $\mathrm{H}$ & -1.49992700 & 2.71132500 & -0.64348700 \\
\hline $\mathrm{H}$ & -0.38905100 & 4.09065800 & -0.75697300 \\
\hline & -1.57439000 & 4.00894600 & 0.54040800 \\
\hline & 0.09629900 & 2.71934500 & 0.79566800 \\
\hline & 0.72496100 & 3.15408300 & 1.94757700 \\
\hline & 0.56015800 & 4.08958200 & 2.45770600 \\
\hline & 1.62377100 & 2.15836500 & 2.31681600 \\
\hline & 2.55115100 & 2.13855200 & 3.47301500 \\
\hline & 2.38879500 & 1.26080300 & 4.10073100 \\
\hline & 2.39479100 & 3.02770800 & 4.08121800 \\
\hline & 3.59324600 & 2.12980900 & 3.14696100 \\
\hline & 2.38252000 & 0.03657800 & -1.25388900 \\
\hline & 3.10375100 & -0.05939800 & -0.10545400 \\
\hline & 0.46727100 & -1.49045000 & 0.31181200 \\
\hline & 1.44155400 & -1.35584900 & 1.25330600 \\
\hline & 0.59162100 & 1.50125200 & 0.49848600 \\
\hline & 1.53154000 & 1.16122600 & 1.42288500 \\
\hline & 2.38032200 & -0.12358800 & 1.25769600 \\
\hline & 0.14657700 & 0.11623000 & -1.12729800 \\
\hline & -2.11345700 & 0.18529600 & -0.34155700 \\
\hline & -3.64894900 & 0.05996400 & 0.00584200 \\
\hline C & -4.37618600 & -0.88104900 & -1.44704800 \\
\hline & -5.71520300 & -1.56521000 & -1.15261700 \\
\hline & -3.65933100 & -1.63450400 & -1.79283200 \\
\hline & -4.48811100 & -0.17782200 & -2.28071100 \\
\hline & -6.09271200 & -2.07094800 & -2.04308900 \\
\hline & -5.61990600 & -2.31710300 & -0.36623100 \\
\hline & -6.47877400 & -0.85239800 & -0.83678200 \\
\hline & -3.63051300 & -0.86680800 & 1.63686600 \\
\hline & -4.92380200 & -0.76742700 & 2.45132300 \\
\hline & -2.79432600 & -0.48052200 & 2.23137500 \\
\hline & -3.39546600 & -1.91817100 & 1.43426000 \\
\hline & -4.84486800 & -1.34853600 & $3.372023 c$ \\
\hline
\end{tabular}




$\begin{array}{lrrr}\text { H } & -5.13874100 & 0.26392700 & 2.73706400 \\ \text { H } & -5.78724000 & -1.14567900 & 1.90116300 \\ \mathrm{C} & -4.24989900 & 1.83180500 & 0.11870600 \\ \mathrm{C} & -5.77504900 & 1.98395500 & 0.09373200 \\ \mathrm{H} & -3.82491500 & 2.39467500 & -0.72022100 \\ \mathrm{H} & -3.84042500 & 2.28121500 & 1.03073300 \\ \mathrm{H} & -6.05600800 & 3.03483600 & 0.18383700 \\ \mathrm{H} & -6.20014400 & 1.61569200 & -0.84207300 \\ \mathrm{H} & -6.25728600 & 1.44729800 & 0.91233500\end{array}$

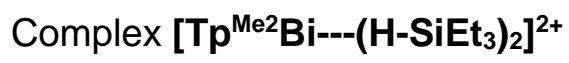

$E_{\text {TOTAL }}=-2206.61692175$ a.u.

$\begin{array}{llll}\text { H } & -4.30202700 & -0.83538700 & 1.12827800 \\ \mathrm{C} & -1.40541700 & 2.45202200 & -2.76487300 \\ \mathrm{H} & -1.46842600 & 1.95029700 & -3.73442500 \\ \mathrm{H} & -0.38145900 & 2.35657900 & -2.39548500 \\ \mathrm{H} & -1.59541900 & 3.51156100 & -2.93623000 \\ \mathrm{C} & -2.40075900 & 1.88887400 & -1.81977600 \\ \mathrm{C} & -3.74717400 & 2.18310900 & -1.68229400 \\ \mathrm{H} & -4.30478500 & 2.92928200 & -2.22520000 \\ \mathrm{C} & -4.24569300 & 1.32013400 & -0.71398200 \\ \mathrm{C} & -5.63214800 & 1.21142300 & -0.19939800 \\ \mathrm{H} & -6.07099300 & 0.24034800 & -0.43732300 \\ \mathrm{H} & -6.25207000 & 1.98186500 & -0.65467800 \\ \mathrm{H} & -5.66931000 & 1.33903700 & 0.88384500 \\ \mathrm{C} & 1.26539000 & 0.01729300 & 2.78213100 \\ \mathrm{H} & 1.74764700 & -0.96194200 & 2.72216300\end{array}$




\begin{tabular}{|c|c|c|c|}
\hline $\mathrm{H}$ & 1.54894500 & 0.46790100 & 3.73253200 \\
\hline $\mathrm{H}$ & 1.66164100 & 0.64673300 & 1.98296700 \\
\hline & -0.20657500 & -0.12280300 & 2.70357400 \\
\hline & -1.13323600 & -0.20519900 & 3.72569600 \\
\hline & -0.93214600 & -0.14397500 & 4.78308900 \\
\hline & -2.37489200 & -0.39229400 & 3.12850300 \\
\hline & -3.69588800 & -0.54935000 & 3.78326800 \\
\hline & -3.58077100 & -0.45676500 & 4.86184700 \\
\hline & -4.13197300 & -1.52741500 & 3.57098600 \\
\hline & -4.40221600 & 0.21204700 & 3.44813100 \\
\hline & -0.26566000 & -3.43178900 & -2.25187600 \\
\hline & 0.64192000 & -2.94402500 & -1.88948000 \\
\hline & -0.42432300 & -3.13852900 & -3.29336100 \\
\hline & -0.08663100 & -4.50691600 & -2.24276800 \\
\hline & -1.45008700 & -3.09920700 & -1.42422700 \\
\hline & -2.61301800 & -3.82511500 & -1.22358400 \\
\hline & -2.84335000 & -4.79985700 & -1.62263100 \\
\hline & -3.42909400 & -3.05137500 & -0.40900900 \\
\hline & -4.78655200 & -3.36161900 & 0.10083000 \\
\hline 1 & -4.82085600 & -3.33620300 & 1.19138000 \\
\hline & -5.08049800 & -4.35774800 & -0.22553100 \\
\hline U & -5.52591600 & -2.65108800 & -0.27419000 \\
\hline & -2.10392000 & 0.90159900 & -0.95631000 \\
\hline & -3.23190000 & 0.54849800 & -0.28798500 \\
\hline & -0.87154400 & -0.24658700 & 1.53713400 \\
\hline & -2.19701500 & -0.41548500 & 1.79937000 \\
\hline A & -1.56205500 & -1.94241500 & -0.74815300 \\
\hline & -2.77388600 & -1.91111200 & -0.13206100 \\
\hline & -3.21987000 & -0.66593900 & 0.66591500 \\
\hline ום & -0.18841000 & -0.17634100 & -0.54568400 \\
\hline $\mathrm{H}$ & 1.78886100 & -1.66700100 & -0.00745000 \\
\hline $\mathrm{H}$ & 0.68895700 & 2.00597800 & 0.13606800 \\
\hline S & 3.23723700 & -2.24289800 & 0.00912500 \\
\hline & 1.42166500 & 3.38993800 & 0.1005310 \\
\hline
\end{tabular}




\begin{tabular}{|c|c|c|c|}
\hline C & 4.32903800 & -0.74830200 & 0.34047300 \\
\hline C & 5.74039000 & -1.07359600 & 0.83801800 \\
\hline $\mathrm{H}$ & 3.82720100 & -0.11131300 & 1.07789000 \\
\hline $\mathrm{H}$ & 4.38405300 & -0.15622900 & -0.58152900 \\
\hline $\mathrm{H}$ & 6.32153400 & -0.16110400 & 0.98524200 \\
\hline $\mathrm{H}$ & 5.71690100 & -1.60030600 & 1.79431100 \\
\hline $\mathrm{H}$ & 6.29066600 & -1.69740000 & 0.13177800 \\
\hline & 3.20512500 & -3.51403200 & 1.38875300 \\
\hline C & 4.33549500 & -4.54570600 & 1.34606800 \\
\hline $\mathrm{H}$ & 2.23845900 & -4.02903500 & 1.34440900 \\
\hline $\mathrm{H}$ & 3.21801200 & -2.98634100 & 2.34997800 \\
\hline $\mathrm{H}$ & 4.24799100 & -5.24908600 & 2.17631300 \\
\hline $\mathrm{H}$ & 4.31148600 & -5.12990500 & 0.42383800 \\
\hline $\mathrm{H}$ & 5.32029000 & -4.08045300 & 1.41730900 \\
\hline C & 3.48691100 & -2.97041900 & -1.70610000 \\
\hline C & 4.94821000 & -3.18451100 & -2.11105200 \\
\hline $\mathrm{H}$ & 3.00929200 & -2.30443700 & -2.43585500 \\
\hline $\mathrm{H}$ & 2.94220300 & -3.91999900 & -1.76224600 \\
\hline $\mathrm{H}$ & 5.01286900 & -3.61853500 & -3.11055500 \\
\hline $\mathrm{H}$ & 5.50122500 & -2.24348000 & -2.12924500 \\
\hline $\mathrm{H}$ & 5.46742900 & -3.86033700 & -1.42939000 \\
\hline C & 2.45927900 & 3.42760000 & 1.66307200 \\
\hline C & 2.97922600 & 4.81730500 & 2.04455800 \\
\hline $\mathrm{H}$ & 3.29740700 & 2.72985500 & 1.54904100 \\
\hline $\mathrm{H}$ & 1.84537000 & 3.03861700 & 2.48321600 \\
\hline $\mathrm{H}$ & 3.57923800 & 4.76725300 & 2.95509600 \\
\hline $\mathrm{H}$ & 3.60698400 & 5.25153300 & 1.26483000 \\
\hline $\mathrm{H}$ & 2.15925400 & 5.51232900 & 2.23532700 \\
\hline 0 & 0.02971800 & 4.64846100 & 0.09886700 \\
\hline C & 0.40748200 & 6.03287900 & -0.43451300 \\
\hline $\mathrm{H}$ & -0.34557600 & 4.72827600 & 1.12574600 \\
\hline & -0.80422700 & 4.24522400 & -0.48573600 \\
\hline & -0.44461500 & 6.71346700 & -0.38323900 \\
\hline & 1.21764700 & 6.48669600 & 0.1389960 \\
\hline
\end{tabular}




$\begin{array}{llll}\mathrm{H} & 0.72780000 & 5.99031700 & -1.47789400 \\ \mathrm{C} & 2.42087600 & 3.30376100 & -1.48916500 \\ \mathrm{C} & 3.56057800 & 4.31983100 & -1.60933000 \\ \mathrm{H} & 1.73669900 & 3.41799300 & -2.33866800 \\ \mathrm{H} & 2.83432300 & 2.29029300 & -1.56987600 \\ \mathrm{H} & 4.07207500 & 4.21600500 & -2.56805300 \\ \mathrm{H} & 3.20104900 & 5.34819700 & -1.54230100 \\ \mathrm{H} & 4.30800200 & 4.17884300 & -0.82603300\end{array}$

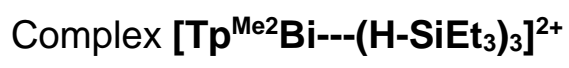

$E_{\text {TOTAL }}=-2734.08805606$ a.u.

$\begin{array}{llll}\text { H } & -4.88780800 & -0.72915800 & 0.48463500 \\ \mathrm{C} & 0.19836100 & -3.39143600 & 1.68697300 \\ \mathrm{H} & 0.51704200 & -3.14050500 & 2.70156300 \\ \mathrm{H} & 0.86323400 & -2.88236700 & 0.98671800 \\ \mathrm{H} & 0.33317300 & -4.46521400 & 1.55605800 \\ \mathrm{C} & -1.22141500 & -3.02468000 & 1.47854000 \\ \mathrm{C} & -2.36157800 & -3.74872800 & 1.78579400 \\ \mathrm{H} & -2.40307200 & -4.73739900 & 2.21367800 \\ \mathrm{C} & -3.44329600 & -2.95202900 & 1.43686900 \\ \mathrm{C} & -4.88993600 & -3.25406300 & 1.56141800 \\ \mathrm{H} & -5.39420800 & -2.53533400 & 2.21002000 \\ \mathrm{H} & -5.01928000 & -4.24649800 & 1.98985700 \\ \mathrm{H} & -5.38816500 & -3.23526000 & 0.59036100 \\ \mathrm{C} & -0.67144000 & 0.16019300 & -3.49178600 \\ \mathrm{H} & -0.81987700 & 0.78945500 & -4.36930800 \\ \mathrm{H} & -0.29964500 & -0.80682000 & -3.83954300 \\ \mathrm{H} & 0.10202100 & 0.62132900 & -2.87500300 \\ \mathrm{C} & -1.94740100 & -0.00954700 & -2.75894600 \\ \mathrm{C} & -3.23304000 & -0.10197800 & -3.26460500 \\ \mathrm{H} & -3.52699800 & -0.02985400 & -4.29932900\end{array}$




\begin{tabular}{|c|c|c|c|}
\hline C & -4.07198900 & -0.31185400 & -2.17857200 \\
\hline C & -5.54543000 & -0.48123700 & -2.17062200 \\
\hline$\vdash$ & -5.92317300 & -0.43135200 & -3.19041200 \\
\hline$\vdash$ & -6.03485400 & 0.30158100 & -1.58811300 \\
\hline 1 & -5.83561600 & -1.44422800 & -1.74671100 \\
\hline 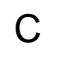 & -0.62263900 & 2.77676300 & 2.45461300 \\
\hline & 0.11083400 & 2.68843800 & 1.65092800 \\
\hline 1 & -0.19928200 & 2.33368700 & 3.35915400 \\
\hline I & -0.77531500 & 3.83828900 & 2.64821800 \\
\hline & -1.90865800 & 2.12781800 & 2.11085900 \\
\hline$C$ & -3.18558100 & 2.43753700 & 2.54804800 \\
\hline H & -3.46475500 & 3.24601800 & 3.20427600 \\
\hline 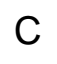 & -4.03806600 & 1.50044500 & 1.97987900 \\
\hline 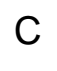 & -5.50864900 & 1.38026400 & 2.13055500 \\
\hline $\mathrm{H}$ & -6.01801400 & 1.44534100 & 1.16746800 \\
\hline r & -5.87666900 & 2.18490400 & 2.76485300 \\
\hline | & -5.78653800 & 0.43065200 & 2.59199200 \\
\hline 1 & -1.60491600 & -1.84384900 & 0.96217000 \\
\hline$\Lambda$ & -2.96370900 & -1.80069400 & 0.93878100 \\
\hline$\Gamma$ & -2.01361200 & -0.15182100 & -1.42316400 \\
\hline$\Gamma$ & -3.31347600 & -0.33892800 & -1.07131700 \\
\hline 1 & -1.99429100 & 1.05301600 & 1.30666700 \\
\hline$\Lambda$ & -3.29616600 & 0.66915800 & 1.23092400 \\
\hline$B$ & -3.71314700 & -0.56267500 & 0.40169500 \\
\hline $\mathrm{Bi}$ & -0.43653000 & -0.09350700 & 0.17333800 \\
\hline r & 0.31568500 & 2.16102500 & -0.76343400 \\
\hline$\vdash$ & 1.07452400 & -1.66259300 & -1.24900900 \\
\hline Si & 0.90431000 & 3.40283500 & -1.49350700 \\
\hline Si & 2.07792200 & -2.57814400 & -2.00002300 \\
\hline & 2.37973900 & 2.73204900 & -2.44581900 \\
\hline C & 2.87487600 & 3.61523900 & -3.59388600 \\
\hline 卜 & 2.12282100 & 1.74181000 & -2.83929000 \\
\hline & 3.18937200 & 2.55953900 & -1.72625300 \\
\hline & 3.74101000 & 3.16681800 & -4.0847 \\
\hline
\end{tabular}




\begin{tabular}{llll} 
H & 2.10511800 & 3.75314500 & -4.35638300 \\
$\mathrm{H}$ & 3.17545600 & 4.60595700 & -3.24887200 \\
$\mathrm{C}$ & -0.51266100 & 4.01661400 & -2.56275800 \\
$\mathrm{C}$ & -0.38869100 & 5.45970600 & -3.05795100 \\
$\mathrm{H}$ & -1.43447600 & 3.90984800 & -1.97877700 \\
$\mathrm{H}$ & -0.62119400 & 3.33697500 & -3.41607600 \\
$\mathrm{H}$ & -1.24739300 & 5.73305700 & -3.67434900 \\
$\mathrm{H}$ & -0.34965000 & 6.16708700 & -2.22706700 \\
$\mathrm{H}$ & 0.50688200 & 5.61173800 & -3.66339300 \\
$\mathrm{C}$ & 1.39594900 & 4.59749900 & -0.12859500 \\
$\mathrm{C}$ & 2.36852000 & 5.69821600 & -0.56129600 \\
$\mathrm{H}$ & 1.84919600 & 4.02220400 & 0.68738000 \\
$\mathrm{H}$ & 0.48399700 & 5.04289100 & 0.28558200 \\
$\mathrm{H}$ & 2.58540800 & 6.37233000 & 0.26968100 \\
$\mathrm{H}$ & 3.32032900 & 5.28201200 & -0.89727800 \\
$\mathrm{H}$ & 1.96771400 & 6.30477600 & -1.37507800 \\
$\mathrm{C}$ & 2.88653800 & -1.47506800 & -3.28977900 \\
$\mathrm{C}$ & 3.57217900 & -2.21239100 & -4.44305700 \\
$\mathrm{H}$ & 3.60592900 & -0.82510000 & -2.77723100 \\
$\mathrm{H}$ & 2.12255100 & -0.80441100 & -3.69724300 \\
$\mathrm{H}$ & 4.02965800 & -1.50544000 & -5.13814200 \\
$\mathrm{H}$ & 4.36207700 & -2.88006300 & -4.09448100 \\
$\mathrm{H}$ & 2.86131100 & -2.81350900 & -5.01378900 \\
$\mathrm{C}$ & 1.00750300 & -3.94908700 & -2.70871400 \\
$\mathrm{C}$ & 1.76026400 & -5.21494300 & -3.12422800 \\
$\mathrm{H}$ & 0.44849600 & -3.54687100 & -3.56215700 \\
$\mathrm{H}$ & 0.25180800 & -4.20350400 & -1.95592600 \\
$\mathrm{H}$ & 1.07458300 & -5.96061300 & -3.53136500 \\
$\mathrm{H}$ & 2.51139000 & -5.01214700 & -3.88962600 \\
$\mathrm{H}$ & 2.27147800 & -5.67412400 & -2.27526200 \\
$\mathrm{H}$ & 3.28740600 & -3.16048700 & -0.68175400 \\
& 4.63052400 & -3.67157400 & -1.20886700 \\
& 2.80456300 & -3.94102300 & -0.08242900 \\
& 3.46440700 & -2.32143200 & 0.00210900 \\
\hline
\end{tabular}




$\begin{array}{llrr}\mathrm{H} & 5.27337300 & -3.99423900 & -0.38733900 \\ \mathrm{H} & 4.50995800 & -4.52461400 & -1.87924300 \\ \mathrm{H} & 5.16906100 & -2.89550500 & -1.75640400 \\ \mathrm{H} & 1.10432000 & -0.20053200 & 2.29173300 \\ \mathrm{Si} & 2.49041200 & 0.03957600 & 2.94247700 \\ \mathrm{C} & 3.47017800 & 0.93163400 & 1.60338200 \\ \mathrm{H} & 3.75171700 & 0.19835300 & 0.83744800 \\ \mathrm{H} & 2.79255300 & 1.63748800 & 1.10512400 \\ \mathrm{C} & 3.15535800 & -1.67104700 & 3.34362500 \\ \mathrm{H} & 2.89765800 & -2.33906000 & 2.51465300 \\ \mathrm{H} & 2.60720700 & -2.04951300 & 4.21472400 \\ \mathrm{C} & 2.16309000 & 1.09758800 & 4.46096800 \\ \mathrm{H} & 2.00610100 & 2.13222100 & 4.13436900 \\ \mathrm{H} & 1.21419900 & 0.76988600 & 4.90168700 \\ \mathrm{C} & 4.66304600 & -1.74876800 & 3.59669100 \\ \mathrm{H} & 4.97671200 & -1.11027500 & 4.42402000 \\ \mathrm{H} & 4.96209300 & -2.76929500 & 3.84409700 \\ \mathrm{H} & 5.23305200 & -1.44910100 & 2.71446600 \\ \mathrm{C} & 3.25862600 & 1.05383400 & 5.52895100 \\ \mathrm{H} & 3.00296900 & 1.69839600 & 6.37220000 \\ \mathrm{H} & 3.39453400 & 0.04492500 & 5.92320300 \\ \mathrm{H} & 4.22237200 & 1.39137300 & 5.14278400 \\ \mathrm{C} & 4.71070200 & 1.68572200 & 2.08737100 \\ \mathrm{H} & 5.43037000 & 1.02098900 & 2.56811000 \\ \mathrm{H} & 5.22546100 & 2.17386100 & 1.25706400 \\ \mathrm{H} & 4.44979100 & 2.46185600 & 2.80982000\end{array}$




\section{References:}

[1] R. J. Errington, Advanced Practical Inorganic and Metal Organic Chemistry, Blackie Academic \& Professional, London, 1997.

[2] W. F. Armarego, C. L. L. Chai, Purification of Laboratory Chemicals, Elsevier, United Kingdom, 2013.

[3] D. Coucouvanis, Inorg. Synth.2002, 33, 220-221.

[4] J. B. Lambert, S. Zhang, C. L. Stern, J. C. Huffman, Science, 1993, 260, 1917-1918.

[5] Frisch, M. J.; Trucks, G. W.; Schlegel, H. B.; Scuseria, G. E.; Robb, M. A.; Cheeseman, J. R.; Scalmani, G.; Barone, V.; Mennucci, B.; Petersson, G. A.; Nakatsuji, H.; Caricato, M.; Li, X.; Hratchian, H. P.; Izmaylov, A. F.; Bloino, J.; Zheng, G.; Sonnenberg, J. L.; Hada, M.; Ehara, M.; Toyota, K.; Fukuda, R.; Hasegawa, J.; Ishida, M.; Nakajima, T.; Honda, Y.; Kitao, O.; Nakai, H.; Vreven, T.; Montgomery Jr., J. A.; Peralta, J. E.; Ogliaro, F.; Bearpark, M. J.; Heyd, J.; Brothers, E. N.; Kudin, K. N.; Staroverov, V. N.; Kobayashi, R.; Normand, J.; Raghavachari, K.; Rendell, A. P.; Burant, J. C.; lyengar, S. S.; Tomasi, J.; Cossi, M.; Rega, N.; Millam, N. J.; Klene, M.; Knox, J. E.; Cross, J. B.; Bakken, V.; Adamo, C.; Jaramillo, J.; Gomperts, R.; Stratmann, R. E.; Yazyev, O.; Austin, A. J.; Cammi, R.; Pomelli, C.; Ochterski, J. W.; Martin, R. L.; Morokuma, K.; Zakrzewski, V. G.; Voth, G. A.; Salvador, P.; Dannenberg, J. J.; Dapprich, S.; Daniels, A. D.; Farkas, Ö.; Foresman, J. B.; Ortiz, J. V.; Cioslowski, J.; Fox, D. J. Gaussian 09, Gaussian, Inc.: Wallingford, CT, USA, 2009.

[6] C. Adamo, V. Barone, J. Chem. Phys. 1999, 110, 6158-6170.

[7] A. Schäfer, C. Huber, R. Ahlrichs, J. Chem. Phys. 1994, 100, 5829-5835. (c) A. Schäfer, H. Horn, R. Ahlrichs, J. Chem. Phys. 1992, 97, 2571-2577.

[8] (a) F. Weinhold, J. Comput. Chem. 2012, 33 (30), 2363-2379; (b) E. D. Glendening, C. R. Landis, F. Weinhold, J. Comput. Chem. 2013, 34 (16), 1429-1437.

[9] F. M. Bickelhaupt, E. J. Baerends, Reviews in Computational Chemistry 2007, Volume 15, 1-86.

[10] G. Te Velde, F. M. Bickelhaupt, E. J. Baerends, C. Fonseca Guerra, S. J. van Gisbergen, J. G. Snijders, T. Ziegler, J. Comput. Chem. 2001, 22, 931-967.

[11] J. G. Snijders, E. J. Baerends, P. Vernooijs, At. Nucl. Data Tables 1982, 26, 483. 
[12] (a) C. Chang, M. Pelissier, Ph. Durand, Phys. Scr. 1986, 34, 394. (b) J.-L. Heully, I. Lindgren, E. Lindroth, S. Lundquist, A.-M. Martensson-Pendrill, J. Phys. B 1986, 19, 2799. (c) E. van Lenthe, R. van Leeuwen, E. J. Baerends, J. G. Snijders, Int. J. Quantum Chem. 1996, 57, 281.

[13] (a) R. F. W. Bader, Atoms In Molecules: A Quantum Theory, Clarendon, Oxford, 1990. (b) The Quantum Theory of Atoms in Molecules [Eds: C. F. Matta, R. J. Boyd] 2007, Wiley-VCH, Weinheim (c) A. D. Becke, K. E. Edgecombe, J. Chem. Phys. 1990, 92, 5397-5403.

[14] T. A. K. AIMAll (Version 16.01.09), TK Gristmill Software, Overland Park KS, USA, 2016 (aim.tkgristmill.com). 